\title{
Cross-Validation with Confidence
}

\author{
Jing Lei ${ }^{1}$ \\ ${ }^{1}$ Department of Statistics, Carnegie Mellon University
}

December 25, 2017

\begin{abstract}
Cross-validation is one of the most popular model selection methods in statistics and machine learning. Despite its wide applicability, traditional cross-validation methods tend to select overfitting models, due to the ignorance of the uncertainty in the testing sample. We develop a new, statistically principled inference tool based on cross-validation that takes into account the uncertainty in the testing sample. This new method outputs a set of highly competitive candidate models containing the best one with guaranteed probability. As a consequence, our method can achieve consistent variable selection in a classical linear regression setting, for which existing cross-validation methods require unconventional split ratios. When used for regularizing tuning parameter selection, the method can provide a further trade-off between prediction accuracy and model interpretability. We demonstrate the performance of the proposed method in several simulated and real data examples.
\end{abstract}

\section{Introduction}

Cross-validation $[27,1,15]$ is one of the most popular methods for model and tuning parameter selection in statistics and machine learning, due to its conceptual simplicity and wide applicability. The basic idea of cross-validation is to fit and evaluate each candidate model on separate data sets so that the performance evaluation is unbiased. Despite its wide use, it is well-known that the traditional cross-validation methods, including the leave-one-out and V-fold variants, tend to select models that over fit. Early theoretical studies of cross-validation $[26,36,35]$ indicate that, under a low-dimensional linear model, cross-validation cannot consistently select the correct model unless the training-testing split ratio tends to zero, which precludes both the leave-one-out and V-fold cross-validation. Although these theory provide deep and important insight for cross-validation procedures, in practice it is very rare to use a small split ratio. In fact, most statistical packages use default split ratios such as nine-to-one (10-fold cross-validation) or four-to-one (5-fold cross-validation). These conventional split ratios remain popular primarily because a small 
split ratio greatly reduces the training sample size and usually results in much less accurate model fitting.

It has been observed in the literature that cross-validation overfits when it fails to take into account the uncertainty in the testing sample. In particular, overfitting occurs if a smaller average test error comes as a result of the random sampling of testing data instead of a truly superior fit. To take into account the testing sample uncertainty and fix the issue of overfitting, we develop a hypothesis testing framework for cross-validation, which we call cross-validation with confidence (CVC). For each candidate model $m$, CVC tests the null hypothesis that the regression functions estimated from model $m$ have the smallest predictive risk among all fitted candidate models, and calculates a $p$-value by comparing the cross-validated residuals of all candidate models. The subset of candidate models for which the null hypotheses are not rejected is a confidence set for model selection.

Depending on the context, cross-validation, and hence CVC, can be used for different purposes. Here we focus on two most common contexts of cross-validation: model selection, and tuning parameter selection for risk minimization.

Model selection. Model selection is concerned with finding the true model, or the one closest to the truth, from a given set of candidate models. Here the set of candidate models is usually discrete and fixed beforehand. For example, the candidate set may consist of all subsets of a given collection of covariates, or all polynomials less than a certain degree. A classical and well studied problem in the cross-validation literature is consistent variable selection in linear regression. Even in the low-dimensional case, cross-validation with a conventional split ratio is known to be inconsistent $[26,36,35]$. We show that the smallest model in the confidence set output by $\mathrm{CVC}$ can achieve consistent variable selection even with a conventional split ratio.

Tuning parameter selection and risk minimization. In many learning problems, the algorithm is indexed by a regularization parameter. Such a tuning parameter can either be continuous, such as the $\lambda$ in the Lasso, or discrete, such as the number of steps in forward stepwise selection. The tuning parameter selection problem is concerned with finding the tuning parameter value, from a given finite candidate set, that leads to the smallest predictive risk. Under this context, cross-validation essentially finds the tuning parameter value whose fitted models have small validated predictive risk. We show that (i) if all the tests in the CVC procedure are conducted at type I error level $\alpha$ for some $\alpha \in(0,1)$, then the confidence set contains the best fitted model with probability at least $1-\alpha$, and (ii) with high probability, the confidence set only contains fitted models that are highly competitive. The main challenge of the testing problem is the high correlation and vastly different scaling between the cross-validated residuals. Our test uses some recent 
results in high-dimensional Gaussian comparison developed by $[7,6]$, which allows us to provide theoretical guarantees for our procedure under weak assumptions.

It is worth noting that risk minimization and model selection are two very different goals, especially when regularization parameter selection is concerned. Regularization typically works by reducing the variance at the cost of adding a small amount of bias, and the added bias may lead to an incorrect model due to the discrete nature of the model selection task. When cross-validation is used to select the regularization parameter, we are often in the context of risk minimization. Without further assumptions, such as that in linear regression the non-zero regression coefficients are sufficiently large and the covariates are weakly correlated, it is generally hard to establish model selection consistency for cross-validation based methods. This is not the focus of the current paper. See [33, 12, 18] for some examples of information criteria based methods that combines regularization parameter tuning and variable selection.

Other related work. The problem of finding a set of plausible candidate models has been studied by several authors. [16] consider this problem for low-dimensional linear regression problems using a hierarchical testing procedure based on the $F$-test. [14] extend and simplify this idea to cover high-dimensional problems, provided that there exists a good variable screening method. [19] develop the "fence" method which finds a set of candidate models that is likely to contain the true model. All these methods only apply to the model selection problem with a fixed candidate set, and the output confidence set of models always includes the saturated model, which may be overfitting.

There are a few heuristic attempts to fix the overfitting issue of cross-validation. For example, [28] propose a heuristic bias correction for $\mathrm{V}$-fold cross-validation, where the average cross-validated risk is offset by the standard error of the $V$ individual subsample cross-validated risk estimates. [22] use a stability-based criterion, which is the ratio between the empirical variance of the fitted vectors and the squared $\ell_{2}$-norm of the average fitted vector. Here the "empirical variance" and "average" are taken over repeated fitting using subsamples. Both methods still output a single candidate model. [20] describe a sample splitting method for assessing variable importance in building predictive regression models, which also uses a hypothesis testing idea. The theoretical properties are not clear for these methods. [34] propose a cross-validation based variable selection procedure which uses a three-way sample split and a least squares fit after Lasso. [13] study model selection consistency of a version of cross-validation specifically designed for penalized likelihood estimators, where, in addition to assumptions on the signal pattern and the design matrix, the split ratio is required to vanish.

Another focus of the cross-validation literature is risk consistency. In an early work, [21] show that cross-validation can select a model with prediction risk close to the optimal candidate model. Similar results in high dimensional regression with Lasso have been 
established recently by $[17,8]$ for $V$-fold cross-validation procedures. [3] study the risk consistency of cross-validated Lasso with a specially designed 2-fold cross-validation variant. The additional value of our CVC method is that it outputs a subset of candidate models with close-to-optimal performance, so that the user has more flexibility in choosing a particular model. For example, one can choose a model that is more parsimonious than the one given by standard cross-validation, or one can pick a model in the confidence set that contains a particularly desirable variable.

Organization of the paper. In Section 2 we briefly review cross-validation and illustrate the cause of overfitting. In Section 3 we present the CVC method and its extensions. In Section 4 we provide general coverage and performance guarantees of the CVC confidence set, and prove variable selection consistency of the CVC method in a classical linear regression setting. Section 5 presents simulated and real data examples. Section 6 concludes the paper with some implementation details and open problems. Appendix A contains technical proofs of main results.

\section{Review of cross-validation}

Suppose we have data $D=\left(X_{i}, Y_{i}\right)_{i=1}^{n}$ independently drawn from a common distribution $P$ on $\mathbb{R}^{p} \times \mathbb{R}$ satisfying

$$
Y_{i}=f\left(X_{i}\right)+\epsilon_{i}
$$

where $f: \mathbb{R}^{p} \mapsto \mathbb{R}$ is an unknown function and $\epsilon$ satisfies $\mathbb{E}(\epsilon \mid X)=0$. We are interested in finding an approximation of $f$ so that we can predict the values of $Y$ for future observations of $X$. Let $\hat{f}$ be an estimate of $f$. We evaluate the quality of $\hat{f}$ by the predictive risk

$$
Q(\hat{f})=\mathbb{E}[\ell(\hat{f}(X), Y) \mid \hat{f}]
$$

where $(X, Y)$ is a future random draw from $P$, and $\ell(\cdot, \cdot)$ is a loss function. A typical example of $\ell(\cdot, \cdot)$ is the squared loss $\ell(\hat{y}, y)=(\hat{y}-y)^{2}$.

\subsection{Validation by sample splitting.}

Here we describe cross-validation using a unified notation for both tuning parameter selection and model selection. Suppose we are given a finite set of candidate models $\mathcal{M}=\{1,2, \ldots, M\}$, where the meaning of each $m \in \mathcal{M}$ can be a model or a tuning parameter value, depending on the context. For each $m \in \mathcal{M}$ one can estimate $f$ by $\hat{f}_{m}$ using an estimating procedure specified by $m$. 
The basic idea of cross-validation originates from estimating the predictive risk using an independent sample. First we split the index set $\{1, \ldots, n\}$ randomly into a training set $I_{\mathrm{tr}}$ and a testing set $I_{\mathrm{te}}=I_{\mathrm{tr}}^{c}$. The data is correspondingly split into a training data $D_{\mathrm{tr}}=\left\{\left(X_{i}, Y_{i}\right): i \in I_{\mathrm{tr}}\right\}$ and a testing data $D_{\mathrm{te}}=\left\{\left(X_{i}, Y_{i}\right): i \in I_{\mathrm{te}}\right\}$. For each candidate model $m$, we obtain an estimate $\hat{f}_{m}$ using $D_{\text {tr }}$. The quality of $\hat{f}_{m}$ is approximated using the empirical average loss on $D_{\text {te }}$ :

$$
\widehat{Q}_{\mathrm{ss}}\left(\hat{f}_{m}\right)=\frac{1}{n_{\mathrm{te}}} \sum_{i \in I_{\mathrm{te}}} \ell\left(\hat{f}_{m}\left(X_{i}\right), Y_{i}\right)
$$

where $n_{\text {te }}$ is the size of $D_{\text {te }}$. Finally, cross-validation chooses the model that gives the smallest empirical risk on testing data:

$$
\hat{m}_{\mathrm{ss}}=\arg \min _{m} \widehat{Q}_{\mathrm{ss}}\left(\hat{f}_{m}\right) .
$$

Here the subscript "ss" stands for sample splitting.

Conditioning on the sample split and the training data $D_{\mathrm{tr}}$, the Law of Large Numbers implies that $\widehat{Q}_{\mathrm{ss}}\left(\hat{f}_{m}\right)$ is close to $Q\left(\hat{f}_{m}\right)$. Therefore, cross-validation approximately minimizes the predictive risk over all candidate estimates $\left\{\hat{f}_{m}: m \in \mathcal{M}\right\}$.

Sources of uncertainty in sample-splitting validation. From (1) we can see that there are two separate sources of uncertainty when using $\hat{m}_{\mathrm{ss}}$ as our estimate of the best $m$. First, the estimates $\hat{f}_{m}$ are obtained from a finite sample $D_{\text {tr }}$, which may not be representative for the overall performance of $\hat{f}_{m}$ when taking into account the randomness of $D_{\mathrm{tr}}$. We call it the estimation uncertainty.

The second source of uncertainty comes from using $\hat{Q}_{\mathrm{ss}}\left(\hat{f}_{m}\right)$ to approximate $Q\left(\hat{f}_{m}\right)$. This is a typical approximation error of sample mean, and the randomness is in the testing data $D_{\text {te. }}$. We call it the validation uncertainty.

\subsection{Reducing the estimation uncertainty: V-fold cross-validation.}

V-fold cross validation extends the idea of sample splitting by repeatedly fitting each candidate model on different subsets of the data. V-fold cross-validation first divides $\{1, \ldots, n\}$ into $V$ equal-sized subsets $I_{1}, \ldots, I_{V}$, and for each $m \in \mathcal{M}$ obtains $\hat{f}_{m}^{(v)}$ using data points in $I_{-v}=I_{v}^{c}$. Then the quality of $m$ as a fitting procedure is approximated by averaging the predictive risk of the estimates:

$$
Q_{\mathrm{cv}}(m)=\frac{1}{V} \sum_{v=1}^{V} Q\left(\hat{f}_{m}^{(v)}\right)
$$


and the model that gives the best estimates is

$$
m_{\mathrm{cv}}^{*}=\arg \min _{m \in \mathcal{M}} Q_{\mathrm{cv}}(m) .
$$

In practice we do not have $Q_{\mathrm{cv}}(m)$ and have to approximate it using sample-split validated predictive risk of $\hat{f}_{m}^{(v)}$ and average over $v=1, \ldots, V$ :

$$
\widehat{Q}_{\mathrm{cv}}(m)=\frac{1}{n} \sum_{v=1}^{V} \sum_{i \in I_{v}} \ell\left(\hat{f}_{m}^{(v)}\left(X_{i}\right), Y_{i}\right)
$$

and the best candidate is estimated by

$$
\hat{m}_{\mathrm{cv}}=\arg \min _{m \in \mathcal{M}} \widehat{Q}_{\mathrm{cv}}(m) .
$$

A key difference between $\mathrm{V}$-fold cross-validation and sample-split validation is that by averaging the approximate predictive risk of $V$ different estimates $\left\{\hat{f}_{m}^{(v)}: 1 \leq v \leq V\right\}$, the $\mathrm{V}$-fold cross-validation provides a more comprehensive assessment by exploring the variability of $\hat{f}_{m}$ due to the randomness of fitting sample. This can be more clearly seen if we re-write $\widehat{Q}_{\mathrm{cv}}$ as follows.

$$
\widehat{Q}_{\mathrm{cv}}(m)=\frac{1}{V} \sum_{v=1}^{V}\left[\left(\frac{n}{V}\right)^{-1} \sum_{i \in I_{v}} \ell\left(\hat{f}_{m}^{(v)}\left(X_{i}\right), Y_{i}\right)\right] \approx \frac{1}{V} \sum_{v=1}^{V} Q\left(\hat{f}_{m}^{(v)}\right),
$$

where each term $(n / V)^{-1} \sum_{i \in I_{v}} \ell\left(\hat{f}_{m}^{(v)}\left(X_{i}\right), Y_{i}\right):=\widehat{Q}_{\mathrm{ss}}\left(\hat{f}_{m}^{(v)}\right)$ is the sample-split validated predictive risk of $\hat{f}_{m}^{(v)}$, which is close to $Q\left(\hat{f}_{m}^{(v)}\right)$ by law of large numbers. While both $\widehat{Q}_{\mathrm{ss}}\left(\hat{f}_{m}\right)$ and $\widehat{Q}_{\mathrm{cv}}(m)$ are unbiased estimate of the overall predictive risk if we use model (or tuning parameter index) $m$ to estimate $f$, the latter often has much smaller variance.

The extreme case of $V=n$ is known as the leave-one-out cross-validation. In modern applications it is often expensive and unnecessary to re-fit the model $n$ times, so most statistical packages use 10 -fold or 5 -fold cross-validation as default choices.

\subsection{Validation uncertainty.}

We start our discussion from a simple example.

Example 1. Let $Y=\mu+\epsilon$ where $\epsilon \sim N(0,1)$. The covariate $X$ is irrelevant here. Let $\mathcal{M}=\{0,1\}$ where $m=0$ corresponds to the model $\mu=0$ so $\hat{f}_{0} \equiv 0$, and $m=1$ corresponds to the model $\mu \in \mathbb{R}$ so $\hat{f}_{1}(x) \equiv \hat{\mu}_{\mathrm{tr}}$ with $\hat{\mu}_{\mathrm{tr}}$ being the training sample mean of $Y$. We consider the squared error loss. Now assume $\mu=0$, then $\hat{f}_{0}$ is always a better estimate than $\hat{f}_{1}$. But

$$
\widehat{Q}\left(\hat{f}_{1}\right)-\widehat{Q}\left(\hat{f}_{0}\right)=\hat{\mu}_{\mathrm{tr}}^{2}-2 \hat{\mu}_{\mathrm{tr}} \bar{\epsilon}_{\mathrm{te}}
$$


where $\bar{\epsilon}_{\mathrm{te}}$ is the testing data sample mean of $\epsilon$. Because $\sqrt{n_{\mathrm{tr}}} \hat{\mu}_{\mathrm{tr}}$ and $\sqrt{n_{\mathrm{te}}} \bar{\epsilon}_{\mathrm{te}}$ are independent standard normal random variables, there is positive probability that $\widehat{Q}\left(\hat{f}_{1}\right)-\widehat{Q}\left(\hat{f}_{0}\right)<0$. This probability becomes larger when $n_{\text {te }}$ is smaller.

In Example 1, cross-validation makes decision based on the sign of $\widehat{Q}\left(\hat{f}_{1}\right)-\widehat{Q}\left(\hat{f}_{0}\right)$, which contains a true signal part $\hat{\mu}_{\mathrm{tr}}^{2}$ and a noise part $-2 \hat{\mu}_{\mathrm{tr}} \bar{\epsilon}_{\mathrm{te}}$. Overfitting happens if the noise overrides the signal. It is clear that in this case it is the uncertainty in the validation step that causes the error, since the estimates $\hat{f}_{0}$ has no uncertainty and we almost surely have $Q\left(\hat{f}_{0}\right)<Q\left(\hat{f}_{1}\right)$.

Since the problem is not due to the estimation error, then intuitively we cannot expect $\mathrm{V}$-fold cross-validation to fix this issue. In fact it is not hard to show that the probability of overfitting is bounded away from 0 for all values of $V$ if $\mathrm{V}$-fold cross-validation is used in Example 1.

In the context of model selection, one way to account for validation uncertainty is to use a training-validation split ratio close to zero. In the context of Example 1, the results in $[26,36,35]$ suggest that $\hat{m}_{\mathrm{ss}}$ is consistent if $n_{\mathrm{tr}} / n \rightarrow 0$. The intuition is that $\bar{\epsilon}_{\mathrm{te}}=O_{P}\left(n_{\mathrm{te}}^{-1 / 2}\right)$, much smaller than $\hat{\mu}$, which has the order $n_{\mathrm{tr}}^{-1 / 2}$.

Although choosing a vanishing training ratio can theoretically address the problem of validation uncertainty for model selection, it is practically undesirable to fit the models with a much smaller sample size. Indeed, the required split ratio cannot be achieved by any conventional V-fold cross-validation. More importantly, using a vanishing training sample ratio may become problematic in the context of tuning parameter selection and risk minimization. A common practice of cross-validation in tuning parameter selection is to apply the fitting procedure on the entire data set with the chosen tuning parameter as a final output. In many important cases, such as bandwidth selection for nonparametric regression [29], and choosing $\lambda$ for the Lasso [9], the optimal tuning parameter may depend on the sample size. Therefore, if $n_{\text {te }} / n \rightarrow 0$, then the optimal $\lambda$ for a training sample of size $n_{\text {te }}$ is unlikely to be a good choice applied to the entire sample.

\section{Cross-validation with confidence}

Now we describe cross-validation with confidence, a procedure that accounts for the validation uncertainty without sacrificing the training sample size. Our discussion starts from the simpler sample-split validation and then extends to the $\mathrm{V}$-fold cross-validation. 


\subsection{Sample-split validation with hypothesis testing}

Here we consider sample-split validation and focus on the conditional inference given the candidate estimates $\left\{\hat{f}_{m}: m \in \mathcal{M}\right\}$ obtained from training data $D_{\mathrm{tr}}$. Recall that we evaluate a fitted model $\hat{f}$ by $Q(\hat{f})=\mathbb{E}(\ell(\hat{f}(X), Y) \mid \hat{f})$. For each $m \in \mathcal{M}$, we consider a hypothesis testing problem

$$
\begin{aligned}
& H_{0, m}: Q\left(\hat{f}_{m}\right) \leq Q\left(\hat{f}_{m^{\prime}}\right) \forall m^{\prime} \neq m \\
& \quad \text { vs } \\
& H_{1, m}: Q\left(\hat{f}_{m}\right)>Q\left(\hat{f}_{m^{\prime}}\right) \text { for some } m^{\prime} \neq m .
\end{aligned}
$$

The hypothesis $H_{0, m}$ means that the fitted model $\hat{f}_{m}$ has the best predictive risk among all fitted models, and $H_{1, m}$ means that there exists another fitted model whose predictive risk is strictly less than $\hat{f}_{m}$.

Let $\alpha \in(0,1)$ be a pre-chosen level of type I error. If we have obtained a $p$-value, denoted by $\hat{p}_{\mathrm{ss}, m}$, for the testing problem (4) for each $m$, the CVC procedure outputs the confidence set

$$
\mathcal{A}_{\mathrm{ss}}=\left\{\hat{f}_{m}: m \in \mathcal{M}, \hat{p}_{\mathrm{ss}, m}>\alpha\right\}
$$

which contains all the fitted models for which $H_{0, m}$ is not rejected.

\subsubsection{Calculating the $p$-values.}

Fix an $m \in \mathcal{M}$, and define random vector $\xi_{m}=\left(\xi_{m, j}: j \neq m\right)$ as

$$
\xi_{m, j}=\ell\left(\hat{f}_{m}(X), Y\right)-\ell\left(\hat{f}_{j}(X), Y\right),
$$

where $(X, Y)$ is a fresh independent sample from the same joint distribution. Let $\mu_{m, j}=$ $\mathbb{E}\left(\xi_{m, j} \mid \hat{f}_{m}, \hat{f}_{j}\right)$. Then the hypotheses in (4) can be written equivalently as

$$
H_{0, m}: \max _{j \neq m} \mu_{m, j} \leq 0, \quad \text { vs } \quad H_{1, m}: \max _{j \neq m} \mu_{m, j}>0 .
$$

(7) is a multivariate mean testing problem. There are a few challenges. First, the dimensionality can be high, as the number of candidate models or tuning parameter values can be on the order of hundreds. Second, the coordinates of $\xi_{m}$ are often highly correlated, since they are calculated from prediction errors of similar models fitted from the same training data and evaluated on the same testing data. Third, the coordinates of $\xi_{m}$ usually have vastly different scaling, depending on the similarity between model $j$ and model $m$. For example, suppose we are considering linear regression with squared error as the loss function, if $\hat{f}_{m}$ and $\hat{f}_{j}$ are both $\sqrt{n}$-consistent then $\xi_{m, j}$ has a variance of order $O\left(n^{-1}\right)$. But if $\hat{f}_{m}$ is $\sqrt{n}$-consistent and $\hat{f}_{j}$ is seriously underfitting (e.g., a linear model missing a 
relevant variable, or Lasso with a very large $\lambda$ ), then the variance of $\xi_{m, j}$ can be as large as a constant.

Considering all these challenges, we derive our test statistic and $p$-value using a recent result of Gaussian comparison and bootstrap of high-dimensional sample means due to [5], which provides an accurate approximation of the maximum of a high-dimensional sample mean using the maximum of a corresponding Gaussian random vector.

The $p$-value $\hat{p}_{\mathrm{ss}, m}$ is calculated as follows, using a studentized Gaussian multiplier bootstrap approach.

1. For $i \in I_{\text {te }}$, let $\xi_{m}^{(i)}$ be the realization of $\xi_{m}$ on the testing data point $\left(X_{i}, Y_{i}\right)$ :

$$
\xi_{m}^{(i)}=\left(\xi_{m, j}^{(i)}: j \neq m\right) \quad \text { where } \xi_{m, j}^{(i)}=\ell\left(\hat{f}_{m}\left(X_{i}\right), Y_{i}\right)-\ell\left(\hat{f}_{j}\left(X_{i}\right), Y_{i}\right)
$$

2. For each $j \neq m$, let $\hat{\mu}_{m, j}$ and $\hat{\sigma}_{m, j}$ be the sample mean and standard deviation of $\left\{\xi_{m, j}^{(i)}: i \in I_{\mathrm{te}}\right\}$.

3. Let $T=\max _{j \neq m} \sqrt{n_{\mathrm{te}}} \frac{\hat{\mu}_{m, j}}{\hat{\sigma}_{m, j}}$.

4. For $b=1, \ldots, B$

(a) Generate iid standard Gaussian random variables $\zeta_{i}, i \in I_{\text {te }}$.

(b) Let

$$
T_{b}^{*}=\max _{j \neq m} \frac{1}{\sqrt{n_{\mathrm{te}}}} \sum_{i \in I_{\mathrm{te}}} \frac{\xi_{m, j}^{(i)}-\hat{\mu}_{m, j}}{\hat{\sigma}_{m, j}} \zeta_{i} .
$$

5. $\hat{p}_{\mathrm{ss}, m}=B^{-1} \sum_{b=1}^{B} \mathbf{1}\left(T_{b}^{*}>T\right)$.

The Gaussian comparison and bootstrap results automatically take into account of the dimensionality and correlation between coordinates of $\xi_{m}$. The studentization addresses the issue of scaling difference. A similar studentized Gaussian approximation has been considered in an unpublished manuscript [6] for different applications.

\subsection{V-fold cross-validation with confidence.}

The $\mathrm{V}$-fold version of the above hypothesis testing procedure is much more complicated, primarily due to the dependence between the fitted models $\hat{f}_{m}^{(v)}$ and the validated predictive $\operatorname{loss} \ell\left(\hat{f}_{m}^{(v)}\left(X_{i}\right), Y_{i}\right)\left(i \in I_{-v}\right)$. 


\subsubsection{A proxy group effect model.}

To further illustrate the issue, the conditional inference for sample-split validation is based on the fact that the validated predictive losses

$$
\left[\ell\left(\hat{f}_{m}\left(X_{i}\right), Y_{i}\right), m=1, \ldots, M\right], \quad i \in I_{\mathrm{te}}
$$

are conditionally independent given $\hat{f}_{1}, \ldots, \hat{f}_{m}$. But in V-fold cross-validation we cannot condition on $\hat{f}_{1}^{(v)}, \ldots, \hat{f}_{m}^{(v)}$ as they are dependent with the data points in $I_{-v}$. More precisely, for $1 \leq i<i^{\prime} \leq n$, let $i \in I_{v}$ and $i^{\prime} \in I_{v^{\prime}}$. There are two possibilities.

Case 1. $v=v^{\prime}$. This is similar to the sample-split case, the dependence between $\ell\left(\hat{f}_{m}^{(v)}\left(X_{i}\right), Y_{i}\right)$ and $\ell\left(\hat{f}_{m}^{(v)}\left(X_{i}\right), Y_{i^{\prime}}\right)$ comes from $\hat{f}_{m}^{(-v)}$. They are conditionally independent given $\hat{f}_{m}^{(v)}$. We denote this conditional distribution of $\ell\left(\hat{f}_{m}^{(v)}\left(X_{i}\right), Y_{i}\right)$ given $\hat{f}_{m}^{(v)}$ as $\hat{\mathcal{F}}_{m}^{(v)}$.

Case 2. $v \neq v^{\prime}$. Now $\ell\left(\hat{f}_{m}^{(v)}\left(X_{i}\right), Y_{i}\right)$ and $\ell\left(\hat{f}_{m}^{\left(v^{\prime}\right)}\left(X_{i^{\prime}}\right), Y_{i^{\prime}}\right)$ depend on each other in that $\left(X_{i}, Y_{i}\right)$ is used as a training sample point in the construction of $\hat{f}_{m}^{\left(v^{\prime}\right)}$, and $\left(X_{i^{\prime}}, Y_{i^{\prime}}\right)$ is used in $\hat{f}_{m}^{(v)}$. Moreover, $\hat{f}_{m}^{(v)}$ and $\hat{f}_{m}^{\left(v^{\prime}\right)}$ share the same $(1-2 / V) n$ out of a total of $(1-1 / V) n$ training sample points.

In the second case described above, $\ell\left(\hat{f}_{m}^{\left(v^{\prime}\right)}\left(X_{i}\right), Y_{i}\right)$ and $\ell\left(\hat{f}_{m}^{(v)}\left(X_{i^{\prime}}\right), Y_{i^{\prime}}\right)$ are not independent samples from $\hat{\mathcal{F}}_{m}^{\left(v^{\prime}\right)}$ and $\hat{\mathcal{F}}_{m}^{(v)}$ due to the dependence between $\left(X_{i}, Y_{i}\right)$ and $\hat{f}_{m}^{\left(v^{\prime}\right)}$ (as well as $\left(X_{i^{\prime}}, Y_{i^{\prime}}\right)$ and $\left.\hat{f}_{m}^{(v)}\right)$. Intuitively, since $\hat{f}_{m}$ is a fitting procedure obtained from $(1-1 / V) n$ sample points, it seems reasonable to believe that the dependence between $\left(X_{i}, Y_{i}\right)$ and $\hat{f}_{m}^{\left(v^{\prime}\right)}$ is weak, so that we can practically ignore such a dependence and treat $\ell\left(\hat{f}_{m}^{\left(v^{\prime}\right)}\left(X_{i}\right), Y_{i}\right)$ and $\ell\left(\hat{f}_{m}^{(v)}\left(X_{i^{\prime}}\right), Y_{i^{\prime}}\right)$ as if they were independent samples from $\hat{\mathcal{F}}_{m}^{\left(v^{\prime}\right)}$ and $\hat{\mathcal{F}}_{m}^{(v)}$, respectively.

Following this intuition, for $1 \leq v \leq V$, let $\hat{\mathcal{F}}^{(v)}$ denote the joint conditional distribution of

$$
\left[\ell\left(\hat{f}_{m}^{(v)}(X), Y\right), 1 \leq m \leq M\right]
$$

given $\left(\hat{f}_{m}^{(v)}, 1 \leq m \leq M\right)$. The we proceed by treating the cross-validated predictive loss

$$
\ell_{i}:=\left[\ell\left(\hat{f}_{m}^{\left(v_{i}\right)}\left(X_{i}, Y_{i}\right)\right), 1 \leq m \leq M\right] \sim \hat{\mathcal{F}}^{\left(v_{i}\right)}
$$

as independent samples from the distributions $\hat{\mathcal{F}}^{\left(v_{i}\right)}$, where $v_{i}$ is the fold id that the $i$ th sample point belongs to. 


\subsubsection{Approximate $p$-value for $\mathrm{V}$-fold CVC.}

Now we have an (approximately) independent sample $\left\{\ell_{i}: 1 \leq i \leq n\right\}$ in $\mathbb{R}^{M}$ of size $n$, where within each group $I_{v}$ the subsample $\left\{\ell_{i}: i \in I_{v}\right\}$ forms an iid sample from $\hat{\mathcal{F}}^{(v)}$. Therefore, we can extend the $p$-value calculation to this case by taking out the group mean effects.

For $m \in \mathcal{M}$, the $\mathrm{V}$-fold version of the hypothesis testing problem becomes:

$$
\begin{aligned}
& H_{0, m}: \frac{1}{V} \sum_{v=1}^{V} Q\left(\hat{f}_{m}^{(v)}\right) \leq \frac{1}{V} \sum_{v=1}^{V} Q\left(\hat{f}_{m^{\prime}}^{(v)}\right), \quad \forall m^{\prime} \neq m \\
& \text { vs } \\
& H_{1, m}: \frac{1}{V} \sum_{v=1}^{V} Q\left(\hat{f}_{m}^{(v)}\right)>\frac{1}{V} \sum_{v=1}^{V} Q\left(\hat{f}_{m^{\prime}}^{(v)}\right) \text { for some } m^{\prime} \neq m .
\end{aligned}
$$

The $p$-values are calculated as follows.

1. Define, for all $j \in \mathcal{M} \backslash\{m\}$,

$$
\xi_{m, j}^{(i)}=\ell\left(\hat{f}_{m}^{\left(v_{i}\right)}\left(X_{i}\right), Y_{i}\right)-\ell\left(\hat{f}_{j}^{\left(v_{i}\right)}\left(X_{i}\right), Y_{i}\right), \quad 1 \leq i \leq n .
$$

2. $\hat{\mu}_{m, j}^{(v)}=\frac{V}{n} \sum_{i \in I_{v}} \xi_{m, j}^{(i)}$, for all $j \neq m, 1 \leq v \leq V$. These are the estimated group mean effect.

3. $\tilde{\xi}_{m, j}^{(i)}=\xi_{m, j}^{(i)}-\hat{\mu}_{m, j}^{\left(v_{i}\right)}$, for all $j \neq m 1 \leq i \leq n$. These are group-wise centered difference of cross-validated predictive loss.

4. Let $\hat{\mu}_{m, j}=V^{-1} \sum_{v=1}^{V} \mu_{m, j}^{(v)}$ be the overall mean effect and $\hat{\sigma}_{m, j}$ be the sample standard deviation of $\left\{\tilde{\xi}_{m, j}^{(i)}: 1 \leq i \leq n\right\}$.

5. Let $T=\max _{j \neq m} \sqrt{n} \frac{\hat{\mu}_{m, j}}{\hat{\sigma}_{m, j}}$.

6. For $b=1, \ldots, B$

(a) Generate iid standard Gaussian random variables $\zeta_{i}, 1 \leq i \leq n$.

(b) Let

$$
T_{b}^{*}=\max _{j \neq m} \frac{1}{\sqrt{n}} \sum_{v=1}^{V} \sum_{i \in I_{v}} \frac{\xi_{m, j}^{(i)}-\hat{\mu}_{m, j}^{(v)}}{\hat{\sigma}_{m, j}} \zeta_{i} .
$$

7. $\hat{p}_{\mathrm{cv}, m}=B^{-1} \sum_{b=1}^{B} \mathbf{1}\left(T_{b}^{*}>T\right)$. 
The level $\alpha$ confidence set of candidate models corresponding to the testing problem (8) is

$$
\mathcal{A}_{\mathrm{cv}}=\left\{m \in \mathcal{M}: \hat{p}_{\mathrm{cv}, m} \geq \alpha\right\} .
$$

The way we compute $\hat{p}_{\mathrm{cv}, m}$ guarantees that the $\mathcal{A}_{\mathrm{cv}}$ is non-empty, and also reveals the relationship between standard cross-validation and CVC.

Proposition 3.1. If $\alpha<0.5$, then

$$
\lim _{B \rightarrow \infty} \mathbb{P}\left(\hat{m}_{\mathrm{cv}} \in \mathcal{A}_{\mathrm{cv}}\right)=1,
$$

where $\hat{m}_{\mathrm{cv}}$ is the standard cross-validation output given in (3).

Proof. Be definition of $\hat{m}_{\mathrm{cv}}$, we always have $T \leq 0$. Because $\alpha<0.5$, the upper $\alpha$ quantile of the maximum of a zero-mean Gaussian vector $\left(T_{b}^{*}\right)$ must be positive. The claim then follows from the Weak Law of Large Numbers.

Following the same reasoning, we can see that among all $m \in \mathcal{M}$, the test statistic $T$ is only non-positive when $m$ is the cross-validation choice $\hat{m}_{\mathrm{cv}}$. Thus, empirically $\hat{m}_{\mathrm{cv}}$ almost always gives the largest $p$-value among all candidate models.

\subsection{Implementation details}

Computational complexity of CVC. The V-fold CVC consists of two main parts. The first part is to split the sample, compute the estimates by holding out each fold, and compute the cross-validated predictive loss for each sample point. This part is also required in ordinary $\mathrm{V}$-fold cross-validation. The additional computing required by CVC is in the second part, where the approximate $p$-values are calculated for each candidate model or tuning parameter value. In this step, for each $m \in \mathcal{M}$, the algorithm will calculate the difference of predictive loss between $m$ and all the other $M-1$ candidates. This requires $O(n(M-1))$ subtractions of two real numbers. The re-centering and standardization costs another $O(n(M-1))$ operations. The multiplier bootstrap with bootstrap sample size $B$ will require $O(n(M-1) B)$ operations. Thus the total additional computing complexity of CVC is $O\left(n M^{2} B\right)$. This additional cost is independent of the dimensionality of the problem. In our numerical experiments, the total running time of CVC is usually a few (less than 5 ) seconds when $n$ is a few hundreds, $M=50$, and $B=200$. This is comparable to other model selection methods based on bootstrap and resampling methods such as the "fence" method [19]. 
Acceleration using inequality selection. The extra computing complexity of CVC has a quadratic dependence on $M$, the total number of candidate models or tuning parameter values. This additional cost can be substantial when $M$ is large. However, when $M$ is large, it is more likely a large proportion of candidate models or tuning parameters will lead to poor estimates. Thus it is possible to eliminate such models quickly without using bootstrap by applying the inequality screening method developed in [6].

More specifically, fix $\alpha^{\prime} \in(0,1)$, and for each $m \in \mathcal{M} j \neq m$, define

$$
\hat{\mathcal{J}}_{m}=\left\{j \in \mathcal{M} \backslash\{m\}: \sqrt{n} \frac{\hat{\mu}_{m, j}}{\hat{\sigma}_{m, j}} \geq-2 \frac{\Phi^{-1}\left(1-\alpha^{\prime} /(M-1)\right)}{\sqrt{1-\left[\Phi^{-1}\left(1-\alpha^{\prime} /(M-1)\right)\right]^{2} / n}}\right\},
$$

where $\Phi$ is the standard normal cumulative distribution function.

Intuitively, the candidates in $\hat{\mathcal{J}}_{m}^{c}$ are "obviously" inferior to $m$ and there is no need to invoke the bootstrap comparison for these models. As a result, one can modify the last three steps of the CVC $p$-value calculation as follows.

\section{CVC $p$-value calculation with inequality selection}

1'-4' The same as steps 1-4 as given in Section 3.2.2.

$5^{\prime}$ Let $T=\max _{j \in \hat{\mathcal{J}}_{m}} \sqrt{n} \frac{\hat{\mu}_{m, j}}{\hat{\sigma}_{m, j}}$.

6' For $b=1, \ldots, B$

(a) Generate iid standard Gaussian random variables $\zeta_{i}, 1 \leq i \leq n$.

(b) Let

$$
T_{b}^{*}=\max _{j \in \hat{\mathcal{J}}_{m}} \frac{1}{\sqrt{n}} \sum_{v=1}^{V} \sum_{i \in I_{v}} \frac{\xi_{m, j}^{(i)}-\hat{\mu}_{m, j}^{(v)}}{\hat{\sigma}_{m, j}} \zeta_{i} .
$$

$7^{\prime} \hat{p}_{\mathrm{cv}, m}=B^{-1} \sum_{b=1}^{B} \mathbf{1}\left(T_{b}^{*}>T\right)$.

In practice, we set $\alpha^{\prime}=\alpha / 10$. In the rare case $\hat{\mathcal{J}}=\emptyset$, which corresponds to the case that $m$ is "obviously" better than all competitors, then we can set $\hat{p}_{\mathrm{cv}, m}=1$. Existing theoretical results (e.g. Theorem 4.5 of [6]) guarantee that, under the same conditions in Corollary 4.2 below, the $p$-value calculated with inequality screening will increase the type I error by no more than $3 \alpha^{\prime}$, provided that $\alpha^{\prime}$ and $\alpha$ are not too small.

\section{Theoretical Properties}

In our theoretical analysis, we first investigate the properties of the confidence sets $\mathcal{A}_{\mathrm{ss}}$ and $\mathcal{A}_{\mathrm{cv}}$ in terms of risk minimization. Then we consider model selection using the CVC 
method, under the context of subset selection for classical linear models.

We always assume that the test sample size is proportional to the total sample size: $n_{\text {te }} / n \in[\delta, 1-\delta]$ for some $\delta \in(0,1 / 2)$. This is satisfied for each fold in the $V$-fold cross-validation with a constant $V$.

\subsection{Quality guarantees of confidence sets.}

\subsubsection{Sample-split validation analysis}

In the analysis of sample-split validation, we condition on the fitted models $\left\{\hat{f}_{m}: m \in \mathcal{M}\right\}$. Our main technical assumption is the tail behavior of the difference of prediction loss $\xi_{m, j}=\ell\left(\hat{f}_{m}(X), Y\right)-\ell\left(\hat{f}_{j}(X), Y\right)$, where the randomness is in the future data pair $(X, Y)$. We assume sub-exponential tail probabilities for standardized $\xi_{m, j}$, characterized by the Orlicz $\psi_{1}$ norm. Given $a \geq 1$, the Orlicz $\psi_{a}$ norm of a random variable $Z$ is

$$
\|Z\|_{\psi_{a}}=\inf \left\{C>0: \mathbb{E} \exp \left(\frac{|Z|}{C}\right)^{a} \leq 2\right\}
$$

Having a finite Orlicz $\psi_{1}$ norm is equivalent to having a sub-exponential tail, and implies finite moments of all orders and exponential concentration of sample mean. A finite Orlicz $\psi_{2}$ norm is equivalent to a sub-Gaussian tail. See [32] and [30] for further discussion on the Orlicz norm.

Following common practice, our analysis of the multiplier bootstrap assumes that $B$ is large enough so the bootstrap sampling variability is negligible. That is, we take $\hat{p}_{\mathrm{ss}, m}$ as the limiting value when $B \rightarrow \infty$.

Theorem 4.1. Let $\mu_{m, j}=\mathbb{E}\left(\xi_{m, j} \mid \hat{f}_{m}, \hat{f}_{j}\right), \sigma_{m, j}^{2}=\operatorname{Var}\left(\xi_{m, j} \mid \hat{f}_{m}, \hat{f}_{j}\right)$. Assume

$$
\sup _{m \neq j}\left\|\frac{\xi_{m, j}-\mu_{m, j}}{\sigma_{m, j}}\right\|_{\psi_{1}} \leq A_{n}
$$

for all $m \neq j$ with

$$
\frac{\left(A_{n} \vee 1\right)^{6} \log ^{7}(M \vee n)}{n^{1-c}} \rightarrow 0,
$$

for some $c \in(0,1)$. The following hold.

1. If $\max _{j \neq m} \frac{\mu_{m, j}}{\sigma_{m, j}} \leq r_{n} \sqrt{\frac{1}{n \log (M \vee n)}}$ for some $r_{n}=o(1)$, then $\mathbb{P}\left(m \in \mathcal{A}_{\mathrm{ss}}\right) \geq 1-\alpha+o(1)$.

2. If $\alpha \in\left(n^{-1}, 1\right)$ and $\max _{j \neq m} \frac{\mu_{m, j}}{\sigma_{m, j}} \geq c^{\prime}\left(A_{n} \vee 1\right) \sqrt{\frac{\log (M \vee n)}{n}}$ for a constant $c^{\prime}$ depending only on $n / n_{\text {te }}$, then $\mathbb{P}\left(m \in \mathcal{A}_{\mathrm{ss}}\right)=o(1)$. 
We prove Theorem 4.1 in Appendix A.1. Part (1) of Theorem 4.1 guarantees the inclusion of competitive estimates. If $\hat{f}_{m}$ is the best estimate in $\left\{\hat{f}_{m}: m \in \mathcal{M}\right\}$, then $H_{0, m}$ is true and $m$ will be included in $\mathcal{A}_{\mathrm{ss}}$ with probability at least $1-\alpha+o(1)$ by just taking $r_{n}=0$ in part (1). In fact, the proof reveals that in the trivial case where $\mu_{m, j}=\mathbb{E}\left(\xi_{m, j} \mid \hat{f}_{m}, \hat{f}_{j}\right)=0$ for all $j \neq m$, we have $\mathbb{P}\left(m \in \mathcal{A}_{\mathrm{ss}}\right)=1-\alpha+o(1)$.

Remark 1. The sub-exponential tail condition (9) can be satisfied, for example, for linear regression with squared loss if the covariate $X$ and noise $\epsilon$ are sub-Gaussian. The Orlicz $\psi_{1}$ norm in (9) will depend on the estimated coefficients $\hat{\beta}_{m}$, which are typically restricted in a bounded set with high probability on $D_{\mathrm{tr}}$.

In practice, the $\psi_{1}$ norm condition of Theorem 4.1 is in general hard to verify exactly. However, given the fact that $\|Z\|_{q} \leq q !\|Z\|_{\psi_{1}}$ for any random variable $Z$, one can estimate $(q !)^{-1}\left\|\left(\xi_{m, m^{\prime}}-\mu_{m, m^{\prime}}\right) / \sigma_{m, m^{\prime}}\right\|_{q}$ for a few small integer values of $q(e . g ., q=1,2,3,4, \ldots)$ using the realized $\xi_{m, m^{\prime}}$ obtained from the validation sample by plugging in $\mu_{m, m^{\prime}}$ and $\sigma_{m, m^{\prime}}$ with empirical estimates. If the estimates do not grow too fast when $q$ grows, then the $\psi_{1}$ norm condition seems plausible. In our simulations, the CVC method works reasonably well in linear regression with noise distribution being student's $t$ with three degrees of freedom.

\subsubsection{Analysis of the group-effect proxy model}

Exact analysis of the V-fold cross-validation is hard due to the dependence between the $\xi_{m, j}^{(i)}$ 's (using the notation in Section 3.2.2) among different folds. Empirically, we found that $\mathrm{V}$-fold CVC has much better power in eliminating suboptimal estimates. We can provide a partial justification by studying the $p$-values $\hat{p}_{\mathrm{cv}, m}$ given in Section 3.2.2 under the group effect model where the dependence are ignored.

The group-effect model as a proxy. In particular, we assume the following model.

A1. $\left(\xi_{m, j}^{(i)}: j \in \mathcal{M} \backslash\{m\}\right)$ are independent across $i=1, \ldots, n$, satisfying $\mathbb{E}\left(\xi_{m, j}^{(i)}\right)=\mu_{m, j}^{(v)}$ and $\operatorname{Var}\left(\xi_{m, j}^{(i)}\right)=\left(\sigma_{m, j}^{(v)}\right)^{2}$ for all $i \in I_{v}$.

A2. There exists a positive number $B_{n}$ such that $B_{n}^{-1} \leq \frac{\sigma_{m, j}^{(v)}}{\sigma_{m, j}^{\left(v^{\prime}\right)}} \leq B_{n}$ for all $m, j, v, v^{\prime}$.

A3. $\left\|\left(\xi_{m, j}^{(i)}-\mu_{m, j}^{(v)}\right) / \sigma_{m, j}^{(v)}\right\|_{\psi_{1}} \leq A_{n}$ for all $m, j$ and all $i \in I_{v}$.

The following corollary is a straightforward extension of Theorem 4.1, providing partial justification of $\mathcal{A}_{\mathrm{cv}}$. 
Corollary 4.2. Under Assumptions A1-A3, if

$$
\frac{\left(A_{n} \vee 1\right)^{6} B_{n}^{6} \log ^{7}(M \vee n)}{n^{1-c}} \rightarrow 0
$$

then the same results of Theorem 4.1 hold for $\mathcal{A}_{\mathrm{cv}}$ with $\mu_{m, j}=V^{-1} \sum_{v=1}^{V} \mu_{m, j}^{(v)}$ and $\sigma_{m, j}^{2}=$ $V^{-1} \sum_{v=1}^{V}\left(\sigma_{m, j}^{(v)}\right)^{2}$.

The only difference from Theorem 4.1 is the involvement of $B_{n}$ in (11). This is necessary because

$$
\left\|\frac{\xi_{m, j}^{(i)}-\mu_{m, j}^{(v)}}{\sigma_{m, j}}\right\|_{\psi_{1}}=\left\|\frac{\xi_{m, j}^{(i)}-\mu_{m, j}^{(v)}}{\sigma_{m, j}^{(v)}}\right\|_{\psi_{1}} \frac{\sigma_{m, j}^{(v)}}{\sigma_{m, j}} \leq A_{n} B_{n} .
$$

\subsection{Model selection consistency in classical linear models.}

Now we show that CVC can be used to overcome the overfitting issue of standard crossvalidation in a classical linear regression setting. Assume that the regression function is linear

$$
Y=X^{T} \beta+\epsilon,
$$

where $X \in \mathbb{R}^{p}$ has covariance $\Sigma$, and $\epsilon$ is independent noise with mean zero and variance $\sigma^{2}$. Here for brevity we assume that $p$ is fixed. The argument and results can be extended to the case that $p$ grows as a small polynomial of $n$ using a union bound argument.

Given a collection of subsets $\mathcal{J}=\left\{J_{1}, \ldots, J_{M}\right\} \subseteq 2^{p}$, we would like to find the $m^{*}$ such that $J_{m^{*}}=\left\{j: \beta_{j} \neq 0\right\}$, assuming that the true model is included in the candidate set. The standard cross-validation (using a single split) estimates $\hat{\beta}_{m}$ by applying a least squares fit using the training data and covariates in $J_{m}$, and evaluates the model by the residual sum of squares on the testing data. CVC works analogously with the same least squares fitting and uses the squared residual as loss function. For any given $\alpha, \mathrm{CVC}$ outputs an confidence set of candidate models. To select the correct model, we consider the most parsimonious model in the confidence set

$$
\hat{m}_{\mathrm{ssc}}=\arg \min _{m \in \mathcal{A}_{\mathrm{ss}}}\left|J_{m}\right|
$$

and

$$
\hat{m}_{\mathrm{cvc}}=\arg \min _{m \in \mathcal{A}_{\mathrm{cv}}}\left|J_{m}\right| .
$$

According to Proposition $3.1, \mathcal{A}_{\mathrm{cv}}$ (and also $\mathcal{A}_{\mathrm{ss}}$ ) is non-empty with high probability when $\alpha<0.5$. So $\hat{m}_{\mathrm{cvc}}$ (and $\hat{m}_{\mathrm{ssc}}$ ) is always well-defined when $\alpha<0.5$.

We consider the following conditions. 
B1. $\lambda_{\min }>0$, where $\lambda_{\min }$ is the minimum eigenvalue of $\Sigma=\mathbb{E}\left(X X^{T}\right)$.

B2. $X$ and $\epsilon$ have finite sixth moments: $\max _{1 \leq j \leq p} \mathbb{E}\left(X_{j}^{6}\right)<\infty, \mathbb{E}\left(\epsilon^{6}\right)<\infty$.

B3. $\max _{1 \leq i \leq n} H_{i i}=O_{P}\left(n^{-1 / 2}\right)$, where $H=X\left(X^{T} X\right)^{-1} X$ is the $n \times n$ hat matrix.

Condition B1 is necessary for the best model to be uniquely defined. Condition B2 requires finite sixth moments for $X$ and $\epsilon$, which is slightly stronger than those in previous work of cross-validation $[36,26]$. We need this in order to control the studentized mean effect in an non-asymptotic manner, which needs $\mathbb{E}\left(\left|\xi_{m, m^{\prime}}\right|^{3}\right)<\infty$, while $\xi_{m, m^{\prime}}$ is a quadratic function of $\epsilon$ and $X$ since we are using the squared loss function. Similar versions of condition B3 have also appeared in previous works. For example, Assumption D of [36] requires $\max _{i} H_{i i}=o(1)$. Our additional rate requirement in B3 is not too stringent. For sub-Gaussian distributions, the maximum diagonal entry of the hat matrix is upper bounded with high probability by $C \log n / n$ for some constant $C$.

Theorem 4.3. Assume model (12) and assumptions B1-B3 hold. Let $\hat{m}_{\mathrm{ssc}}$ be given as in (13) with $\mathcal{A}_{\mathrm{ss}}$ being the sample split validation confidence set using type I error level $\alpha_{n} \rightarrow 0$ and $\alpha_{n}>n^{-1}$. Then $\mathbb{P}\left(\hat{m}_{\mathrm{ssc}}=m^{*}\right) \rightarrow 1$.

We prove Theorem 4.3 in Appendix A.2. One challenge is to show that $\hat{f}_{m^{*}}$ has the smallest risk with high probability, which involves the Kolmogorov-Rogozin anti-concentration inequality [25] since we do not assume Gaussianity for $X$ or $\epsilon$.

Corollary 4.4. Under the same conditions in Theorem 4.3. Let $\hat{m}_{\mathrm{cvc}}$ be given as in (14) using type $I$ error level $\alpha_{n} \rightarrow 0$ and $\alpha_{n}>n^{-1}$. Then $\mathbb{P}\left(\hat{m}_{\mathrm{cvc}}=m^{*}\right) \rightarrow 1$.

Remark 2. Unlike Corollary 4.2, which is proved under a proxy group effect model, Corollary 4.4 is established for the genuine CVC procedure without ignoring the dependence between the validated predictive loss across different folds. This is possible since we do not ask for exact type I error control but simply a vanishing type I error probability.

Remark 3 (Choice of $\alpha_{n}$ in practice). Our theory requires $\alpha_{n}$ to vanish as $n$ grows in order to control the probability of not including the best model. But this is necessary only if all candidate models are equally good. In practice it is often the case that the best model is strictly better than other candidate models, so the true type I error is smaller than the nominal level. In practice we recommend using CVC with $\alpha_{n}=0.05$, the traditional level of statistical significance. All numerical examples in this paper are conducted with $\alpha=0.05$.

\section{$5 \quad$ Numerical experiments}

We illustrate the performance of the CVC method using synthetic and real data sets. All cross-validation methods, including CVC are implemented using 5 folds. CVC is 
implemented using inequality screening with $\left(\alpha, \alpha^{\prime}\right)=(0.05,0.005)$ and $B=200$.

\subsection{Simulation 1: Subset selection consistency in linear models.}

In this simulation we demonstrate the model selection consistency of CVC to support the theory developed in Section 4.2. We adopt the synthetic data set in [26], where the covariate $X$ has five coordinates including intercept, with a sample size $n=40$ (see Table 1 of [26] for the complete data set). The response variable $Y$ is generated by the linear model (12) with a pre-determined $\beta$ and independent noise $\epsilon$. We consider two true models $\beta^{T}=(2,0,0,4,0)$ and $(2,9,0,4,8)$ that correspond to a sparse model and a less sparse model. The set of candidate models consists of all 16 possible models that include the intercept term. Two noise distributions are experimented: standard normal and student's $t$-distribution with three degrees of freedom. For each type of noise distribution, we consider two noise-to-signal ratios. The first one uses standard Gaussian and student's $t(3)$ to generate the noises. The second one amplifies the noises by a factor of 2 .

The sample size $n=40$ is far from the regime of model selection consistency. To illustrate the effect of sample size, we generate additional sample points with $X$ drawn from a multivariate Gaussian distribution whose parameters are given by the sample mean and covariance of the original data set. We consider varying sample sizes between $n=40$ and $n=640$, where $n=40$ corresponds to the original data set. We compare the performance of five methods, the ordinary cross-validation (cv), the most parsimonious model in $\mathcal{A}_{\mathrm{cv}}$ (cvc), BIC, the confidence set of models using $F$-test proposed by [14], and the "fence" method [19].

Figures 1 and 2 summarize the frequency each method correctly selects the model, and the number of models that are contained in the confidence set, over 100 independently generated data set. For a very small sample size $n=40$, the CVC method does not perform as well as the other methods, as it often selects the underfitting models. The performance of CVC increases rapidly as the sample size $n$ increases, with a perfect rate of correct selection as soon as $n$ reaches 320 . Meanwhile, the performance of the standard cross-validation does not improve as $n$ increases, with rates of correct selection staying away from 1 . This agrees with the theory that CVC can consistently select the true model, while the standard cross-validation tends to overfit as long as the training sample ratio stays constant.

Overall, CVC is highly competitive when $n$ is moderately large and the true model is sparse. Moreover, except in the high noise case with student's $t(3)$ noise, the CVC confidence set tends to be smaller than the F-test based confidence set [14] when $n$ is moderately large. 

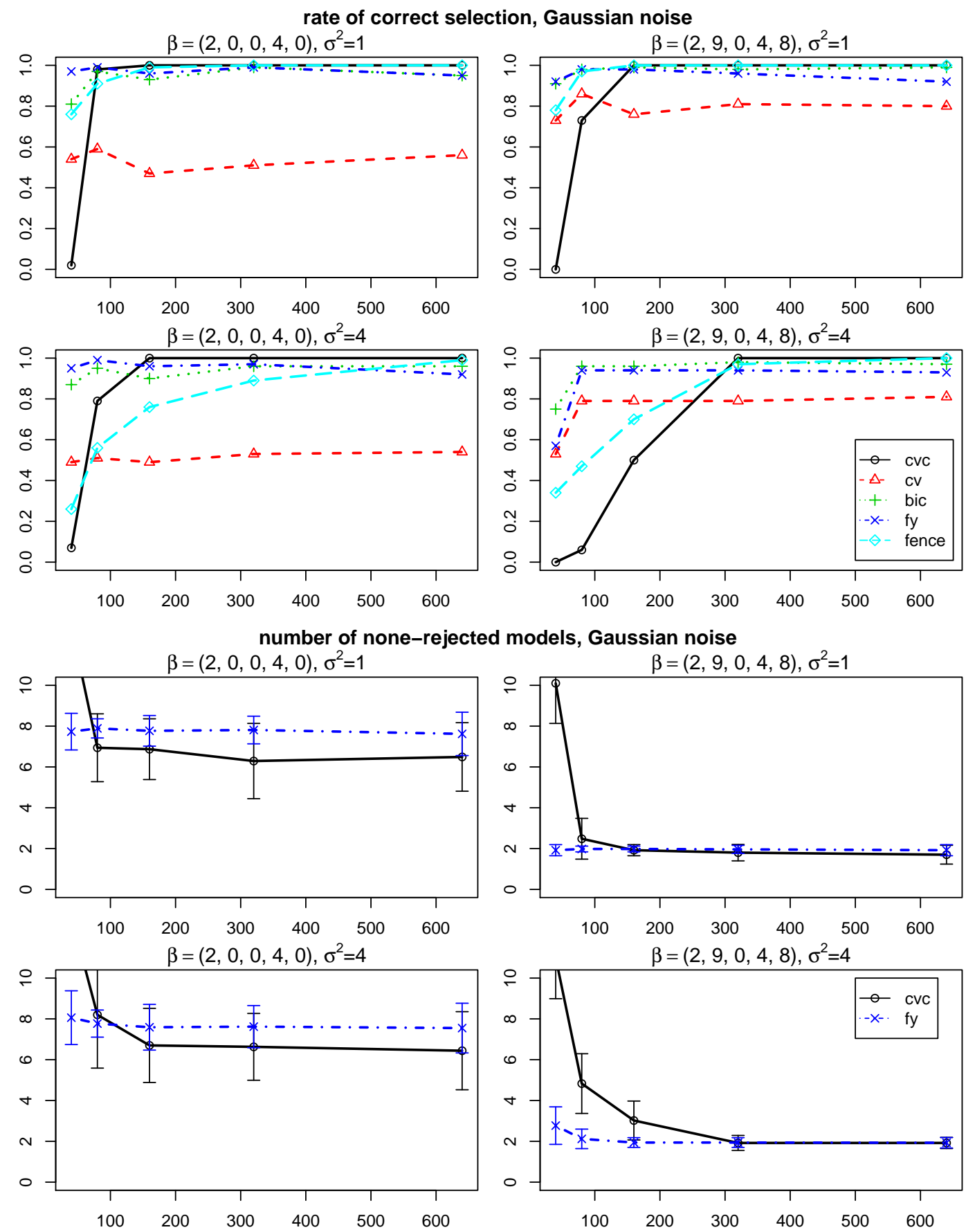

Figure 1: Simulation 1: linear regression with $p=5$ and Gaussian noise. First two rows: rate of correct subset selection as a function of sample size. Bottom two rows: number of none-rejected candidate models as a function of sample size. 

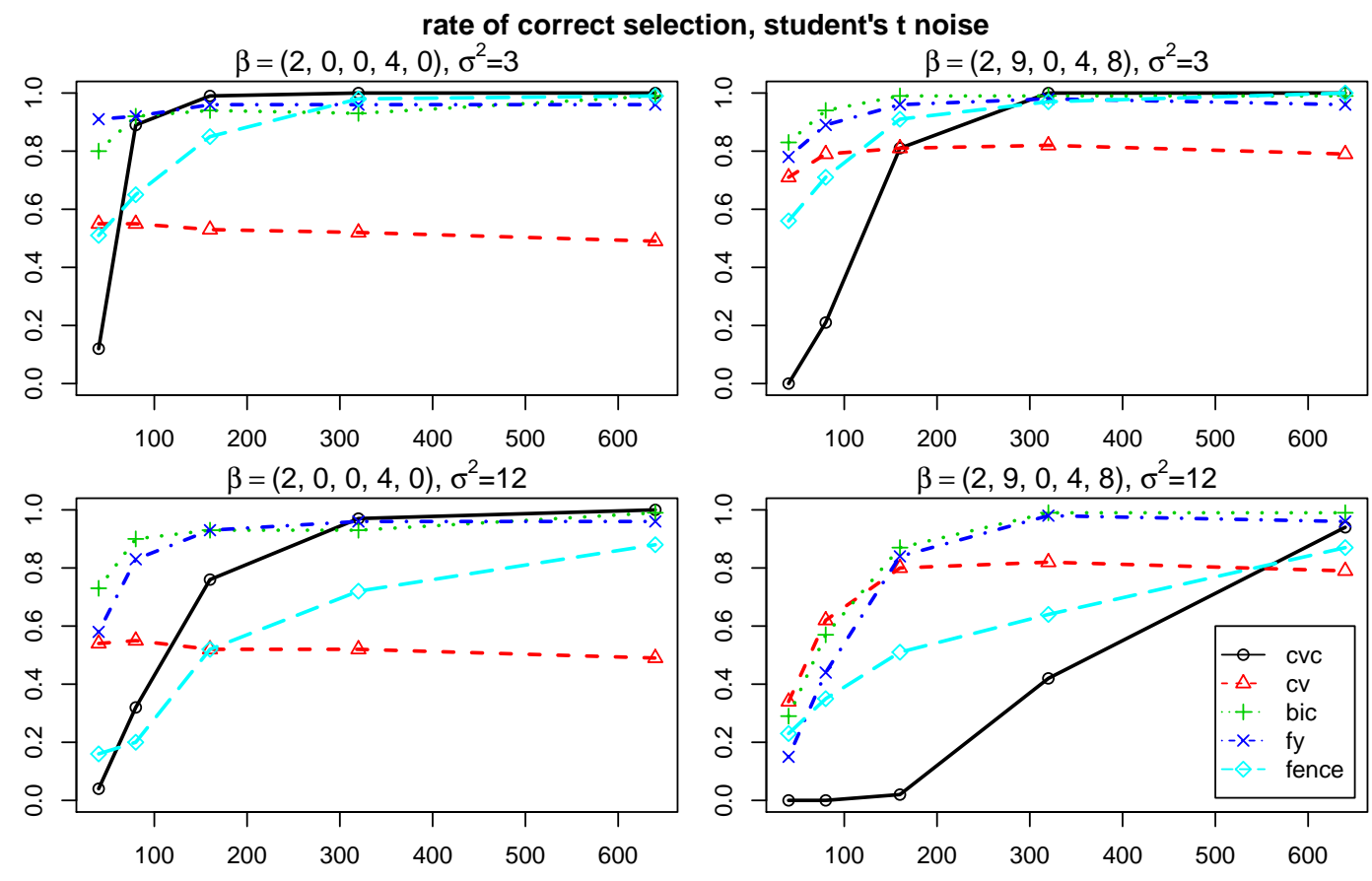

number of none-rejected models, student's t noise

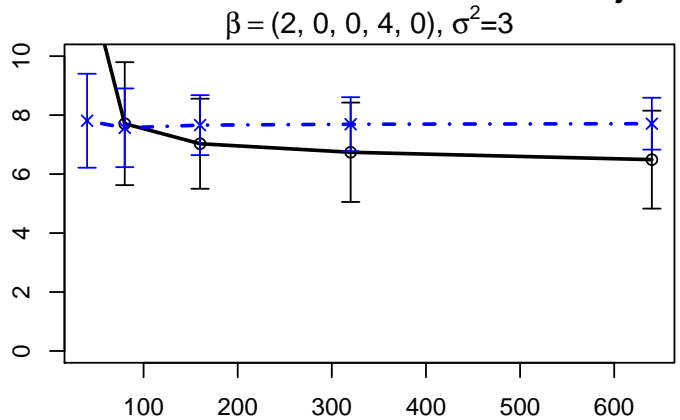

$\beta=(2,9,0,4,8), \sigma^{2}=3$
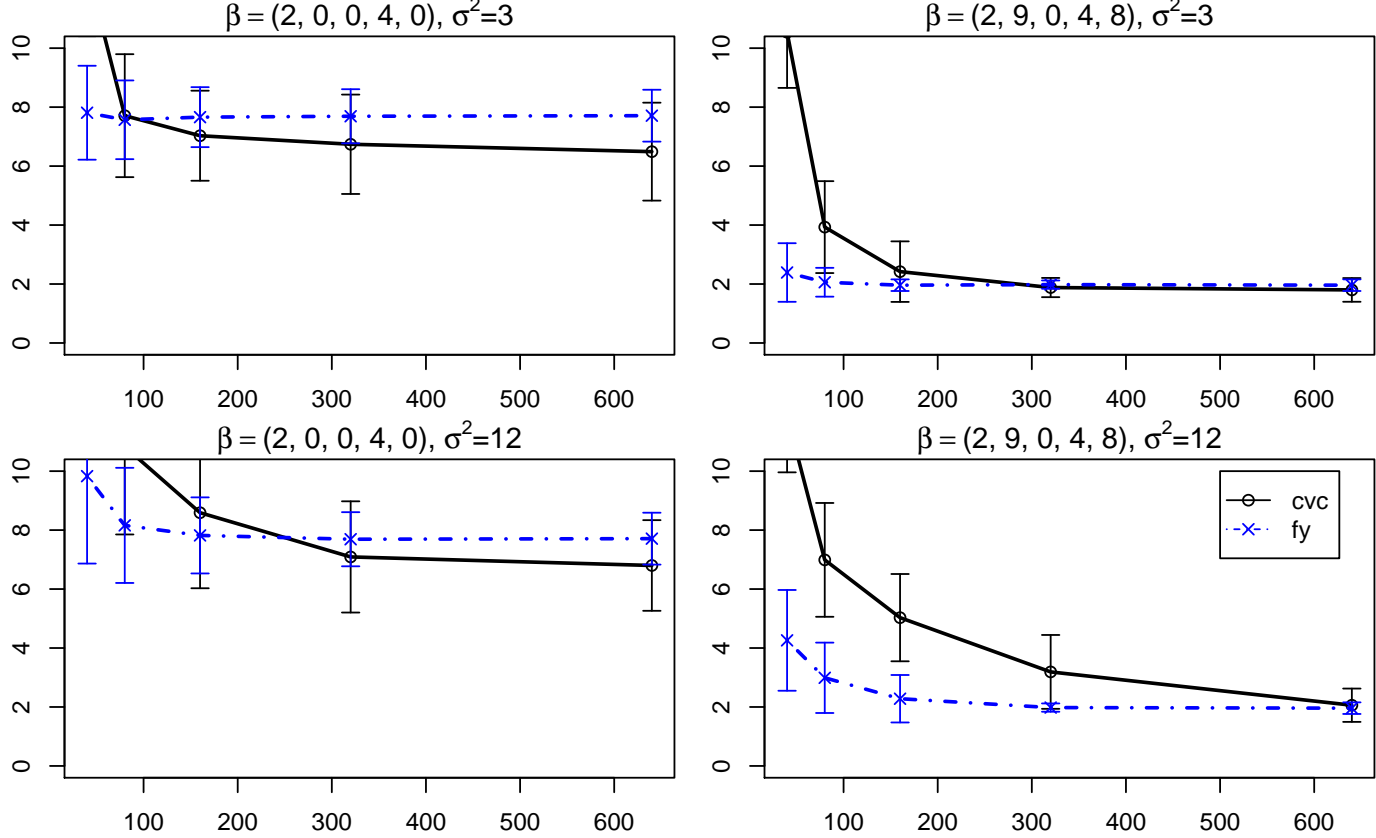

Figure 2: Simulation 1: linear regression with $p=5$ and student's $t_{3}$ noise. First two rows: rate of correct subset selection as a function of sample size. Bottom two rows: number of none-rejected candidate models as a function of sample size. 


\subsection{Simulation 2: Tuning the Lasso for risk minimization}

We use this simulation to demonstrate the performance of CVC in choosing the Lasso tuning parameter for predictive risk minimization. We generate data from a linear regression model (12), where $X \in \mathbb{R}^{200}$ has a multivariate Gaussian distribution $N(0, \Sigma)$, and $\epsilon$ is an independent standard Gaussian noise. We consider the squared loss $\ell(\hat{y}, y)=(\hat{y}-y)^{2}$. For any estimate $\hat{\beta}$ of $\beta$, the linear model leads to a closed-form formula for $Q(\hat{\beta})$ :

$$
Q(\hat{\beta})=\mathbb{E}\left[\left(Y-X^{T} \hat{\beta}\right)^{2} \mid \hat{\beta}\right]=(\hat{\beta}-\beta)^{T} \Sigma(\hat{\beta}-\beta)+1 .
$$

We consider two settings of $\Sigma$. In the identity setting, $\Sigma$ is the $200 \times 200$ identity matrix. In the correlated setting, the diagonal entries of $\Sigma$ are 1 and off-diagonal entries are 0.5 . We also consider two settings of $\beta$. In each setting, the first $s$ coordinates of $\beta$ take value \pm 1 with randomly chosen signs, and the next $s$ coordinates are generated from $N\left(0, I_{s}\right)$, and the remaining coordinates are 0 . In the sparse setting, we set $s=5$, while in the dense setting, we set $s=25$ so that a quarter of the coordinates of $\beta$ are non-zero.

For each generated data set we first obtain a sequence of 50 tuning parameters using $\mathrm{R}$ package glmnet on the whole data set. Using these 50 candidate values of $\lambda$, we compare four methods: the original $V$-fold cross-validation ("cv"), the 1-standard-error rule ["1se", 28], the estimation stability method ["es", 22], and the most parsimonious model in the CVC confidence set with $\alpha=0.05$ ("cvc"), defined as

$$
\hat{\lambda}_{\mathrm{cvc}}=\max \left\{\lambda: \lambda \in \mathcal{A}_{\mathrm{cv}}\right\} \text {. }
$$

We run the simulation with sample size $n=200$, and repeat 100 times for each combination of $\beta$ and $\Sigma$.

Evaluation and comparison of cross-validation based methods. In the context of risk minimization using cross-validation, a common practice is to first find a promising value of tuning parameter (such as the one given by cross-validation or its variants) and then obtain a final estimate using the entire data set and the selected tuning parameter value. This approach has been used in some existing modifications of cross-validation, including the 1-standard-error rule [28] and the stability rule [22]. In the case of CVC and Lasso, we argue that one must take into account the sample size difference between the training sample used in cross-validation and the entire sample. More precisely, in V-fold cross-validation, each candidate value of tuning parameter is validated based on the estimates obtained from a training sample of size $n\left(1-V^{-1}\right)$, but the final estimate is obtained on a training sample of size $n$. Intuitively, the optimal value of tuning parameter for one sample size is not necessarily also optimal for a different sample size, because when the sample size gets larger, less regularization is required. In fact, it has been suggested in the Lasso literature 

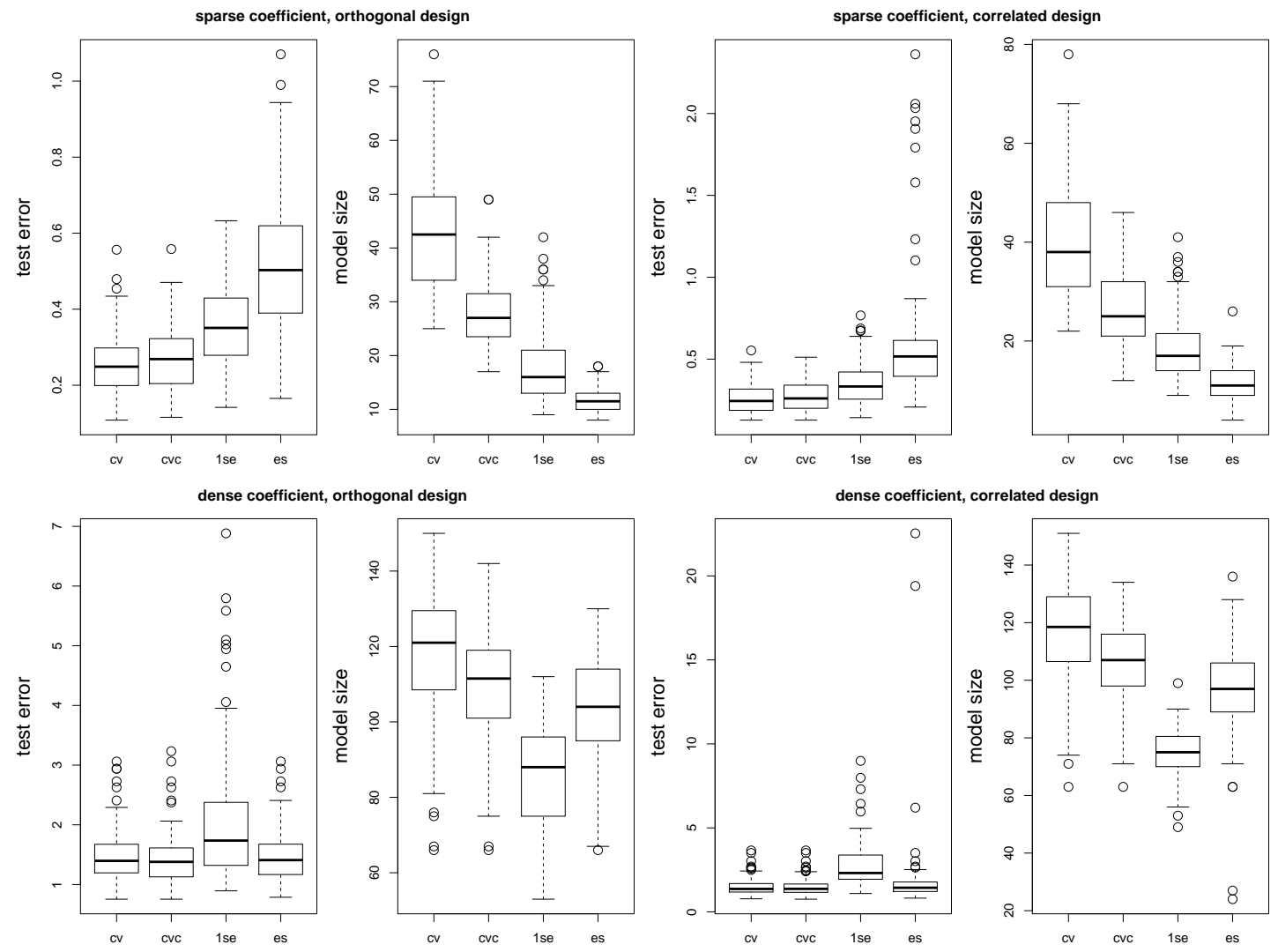

Figure 3: Risk and model size of four methods of Lasso tuning parameter selection. Top row: sparse model. Bottom row: dense model. Columns 1-2: orthogonal design. Columns 3-4: correlated design.

that the optimal value of $\lambda$ for both prediction and estimation is inversely proportional to the square root of the training sample size $[2,31,23,9]$. Therefore, in our implementation of CVC we make use of this observation by obtaining the final estimate on the entire data set with tuning parameter value $\lambda=\sqrt{1-V^{-1}} \hat{\lambda}_{\mathrm{cvc}}$.

Figure 3 summarizes the performance. We can see that the CVC method offers highly competitive prediction accuracy comparing to standard cross-validation, while using fewer predictors. Both CVC and ES have good adaptivity to the model sparsity, using more predictors when the model is dense. Overall, CVC provides a better trade-off between interpretability and predictive accuracy compared to other methods. Moreover, the median value of $\mathcal{A}_{\mathrm{cv}}$ is between 4 and 5 for all settings, and the $\mathcal{A}_{\mathrm{cv}}$ covers the best tuning parameter value with a frequency almost exactly 0.95 in all settings. 


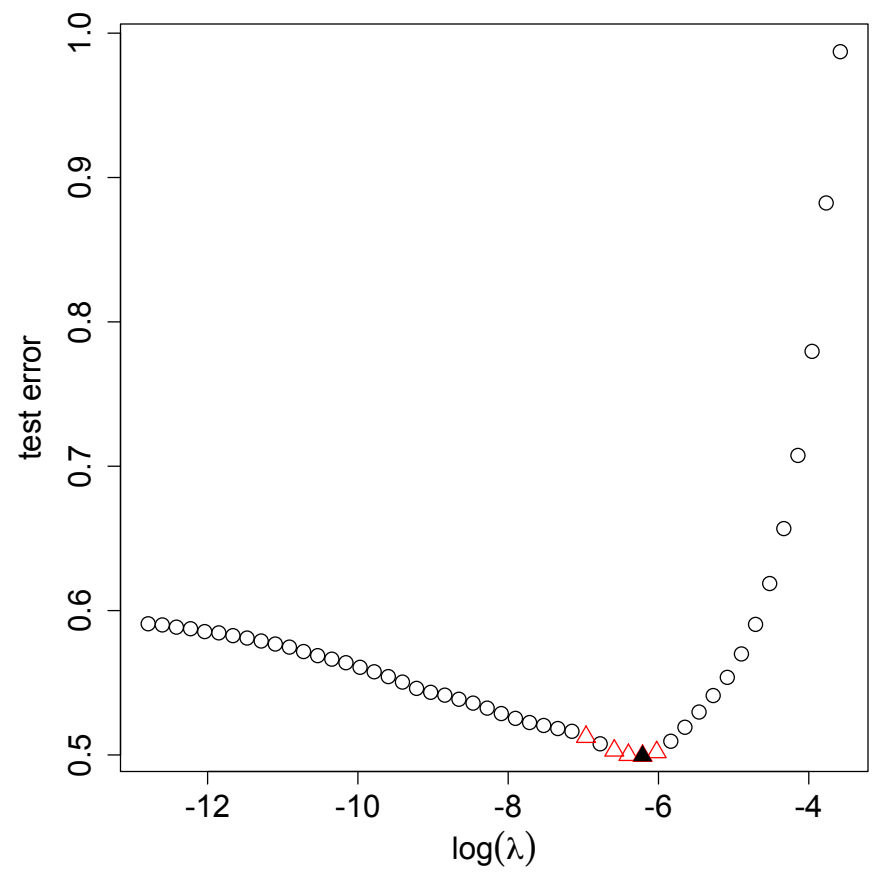

Figure 4: Diabetes data example. The cross-validation test errors for 50 values of $\lambda$. The triangles correspond to the $\lambda$ values in $\mathcal{A}_{\mathrm{cv}}$ given by 5 -fold CVC with $\alpha=0.05$. The solid triangle corresponds to the $\lambda$ chosen by standard 5 -fold cross-validation.

\subsection{The diabetes data example.}

We apply the CVC method to the diabetes data, which has been used in [11] to illustrate the LARS algorithm in sparse linear regression. The data contains $n=442$ sample points, with ten covariates including age, sex, body mass index, blood pressure, and six blood serum measurements. If we consider all the quadratic terms as in the literature, there will be 64 predictors (the sex variable is coded as $\{0,1\}$ and has no square term). The response variable is a quantitative measure of diabetes progression one year after the covariates are measured. Following common practice, we center and standardize all variables.

Figure 4 shows the result of 5 -fold CVC with $\alpha=0.05$ on the full data set. The base estimator is the Lasso, with 50 equally spaced values (on the log scale) of the penalty parameter $\lambda$. We plot the cross-validated test errors for all candidate values of $\lambda$. The triangle points correspond to the tuning parameter values in $\mathcal{A}_{\mathrm{cv}}$, and the solid triangle corresponds to the $\lambda$ chosen by standard 5 -fold cross-validation. 5-fold CVC outputs five candidate values of $\lambda$, one of which gives a very close test error as the standard cross-validation but a more parsimonious model fit. 

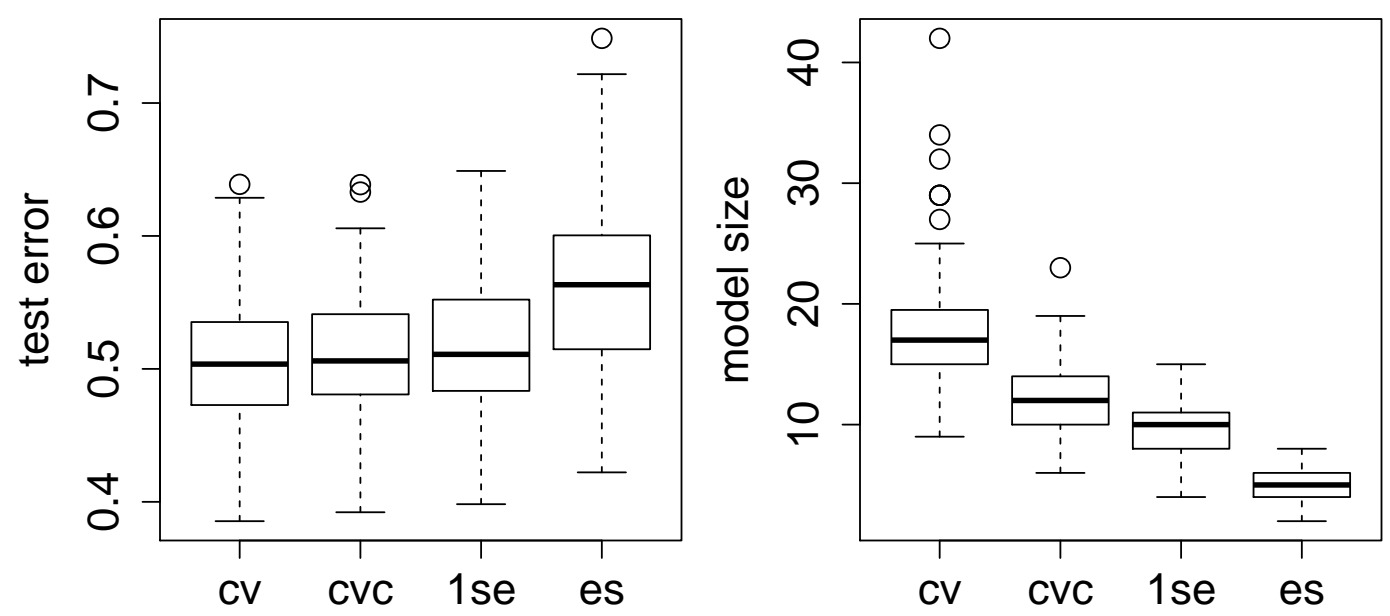

Figure 5: Diabetes data example. Box-plots of hold-out test errors and number of selected variables over 100 repeated hold-out splitting. Compared estimators are the standard cross-validation ("cv"), the most parsimonious model in $\mathcal{A}_{\mathrm{cv}}$ ("cvc"), the 1-standard-error rule ("1se"), and the estimation stability rule ("es").

To investigate the predictive performance of the final estimate, we split the data so that 300 sample points are used for estimation, including implementing cross-validation or CVC, and the remaining 142 sample points are held out for test error calculation. To be specific, all competing methods (cv, cvc, 1se, es) are based on 5-fold cross-validation in picking $\lambda$, and then obtain the final estimate of regression coefficient using Lasso with the chosen $\lambda$ on the full training sample of 300 data points. As we explained in the previous subsection, the CVC method uses a scaled value of $\lambda$ to offset the training sample size difference when obtaining the final estimate. Then we use the untouched set of 142 hold-out sample points to calculate the test error for the final estimate. We repeat this experiment 100 times, each time with an independent hold-out split.

The results are summarized in Figure 5. Comparing to the simulation results in Section 5.2, the diabetes data set is more similar to a low-dimensional model, where more regularization can be used for better interpretation without losing prediction accuracy. We see that the most parsimonious CVC model gives very close test error as the standard cross-validation, but has much fewer predictors. The 1se method is also very competitive, with even more interpretable estimates and a slightly larger predictive risk. 


\section{Discussion}

The wide applicability of cross-validation makes it worthwhile to further extend our understanding of CVC under other contexts. Several extensions shall be pursued in future work. First, it would be interesting to study and extend CVC for high dimensional regression model selection problems. Second, in our consistency analysis of sparse linear regression in Section 4.2, we considered independent noise. If the response variable is binary, then we need to extend the theory to cover heteroskedastic noises. Moreover, it is also possible to extend the framework of CVC to unsupervised learning problems. For example, in $K$-means or model-based clustering, one needs to specify the number of clusters. Given a set of estimated clusters, the value of loss function at a test sample point can be set as the squared distance to the closest cluster center. Similar extensions can also be made in low rank matrix approximation using singular value decompositions [24] and network community detection [4], where various forms of cross-validation can be used for model selection.

\section{A Proofs}

\section{A.1 Proof of Section 4.1}

In the construction of our $p$-value $\hat{p}_{m}$, the main test statistic is the maximum of the studentized sample mean of $\left(\xi_{m}^{(i)}: i \in I_{\mathrm{te}}\right)$. The reference distribution is the maximum of a Gaussian vector with covariance $\hat{\Gamma}_{m}$, the sample correlation matrix of $\left(\xi_{m}^{(i)}: i \in I_{\mathrm{te}}\right)$. Therefore, the main task is to prove that the maximum of a studentized sample mean has similar distribution as the maximum of a Gaussian random vector with same covariance structure. A result of this type is given as part of the proof of Theorem 4.3 of [6]. Here we provide a self-contained proof using more compact notation and under the assumption of bounded Orlicz $\psi_{2}$ norm for $\left(\xi_{m, j}-\mu_{m, j}\right) / \sigma_{m, j}$. Finally, the unnumbered constants $c, c^{\prime}$ may vary from line to line. Numbered constants, $c_{1}, c_{2}, c_{3}, C_{1}, C_{2}$, etc, correspond to those specified in corresponding theorems.

Additional notation. Here we fix $m$ and will drop $m$ in the notation. Let $\mu, \Sigma, \Gamma$ be the mean, covariance and correlation matrix of the $M-1$ dimensional random vector $\xi=\left(\xi_{m, j}: j \neq m\right)$ (conditional on $D_{\text {tr }}$ ). The corresponding sample versions are $\hat{\mu}, \hat{\Sigma}$, and $\hat{\Gamma}$. Let $\sigma_{j}^{2}$ be the $j$ th diagonal element of $\Sigma$, and $\hat{\sigma}_{j}$ the sample version. Finally, let $N=M \vee n$.

Lemma A.1. Let $W_{1}, W_{2}$ be random variables, then

$$
\left\|W_{1} W_{2}\right\|_{\psi_{1}} \leq\left\|W_{1}\right\|_{\psi_{2}}\left\|W_{2}\right\|_{\psi_{2}}
$$


Proof.

$$
\begin{aligned}
& \left\|\frac{W_{1} W_{2}}{\left\|W_{1}\right\|_{\psi_{2}}\left\|W_{2}\right\|_{\psi_{2}}}\right\|_{\psi_{1}} \leq\left\|\frac{W_{1}^{2}}{2\left\|W_{1}\right\|_{\psi_{2}}^{2}}+\frac{W_{2}^{2}}{2\left\|W_{2}\right\|_{\psi_{2}}^{2}}\right\|_{\psi_{1}} \\
& \leq \frac{1}{2}\left\|\frac{W_{1}^{2}}{\left\|W_{1}\right\|_{\psi_{2}}^{2}}\right\|_{\psi_{1}}+\frac{1}{2}\left\|\frac{W_{2}^{2}}{\left\|W_{2}\right\|_{\psi_{2}}^{2}}\right\|_{\psi_{1}}=1 .
\end{aligned}
$$

Lemma A.2. There exist universal positive constants $c_{1}, c_{2}$ such that if (9) holds with $\left(A_{n}^{2}+1\right) \leq c_{1} \sqrt{n / \log N}$ then the following holds.

$$
\begin{gathered}
\mathbb{P}\left[\max _{j}\left|\frac{\hat{\mu}_{j}-\mu_{j}}{\sigma_{j}}\right| \geq c_{2} A_{n} \sqrt{\log N / n}\right] \leq N^{-1} . \\
\mathbb{P}\left[\max _{j}\left|\frac{\hat{\sigma}_{j}^{2}}{\sigma_{j}^{2}}-1\right| \geq c_{2} A_{n}^{2} \sqrt{\log N / n}\right] \leq N^{-1} . \\
\mathbb{P}\left[\max _{j, j^{\prime}}\left|\hat{\Gamma}_{j, j^{\prime}}-\Gamma_{j, j^{\prime}}\right| \geq c_{2} A_{n}^{2} \sqrt{\log N / n}\right] \leq N^{-1} .
\end{gathered}
$$

Proof of Lemma A.2. Since a smaller $A_{n}$ means better concentration, without loss of generality we can assume that $A_{n} \geq 1$.

The first inequality directly follows from the fact that $\|Z\|_{\psi_{1}} \leq\|Z\|_{\psi_{2}}$, the Bernstein's inequality using Orlicz norm (Lemma 2.2.11 of [32]), and union bound.

For the second inequality, note that

$$
\left\|\left|\left(\frac{\xi_{j}-\mu_{j}}{\sigma_{j}}\right)^{2}-1\right|^{1 / 2}\right\|_{\psi_{1}} \leq\left\|\frac{\xi_{j}-\mu_{j}}{\sigma_{j}}\right\|_{\psi_{1}}+\|1\|_{\psi_{1}}=A_{n}+(\log 2)^{-1} .
$$

We can re-write $\hat{\sigma}_{j}^{2} / \sigma_{j}^{2}-1$ as

$$
\frac{\hat{\sigma}_{j}^{2}-\sigma_{j}^{2}}{\sigma_{j}^{2}}=n_{\mathrm{te}}^{-1} \sum_{i \in I_{\mathrm{te}}}\left[\left(\frac{\xi_{j}^{(i)}-\mu_{j}}{\sigma_{j}}\right)^{2}-1\right]-\left(\frac{\hat{\mu}_{j}-\mu_{j}}{\sigma_{j}}\right)^{2} .
$$

Then the claim follows by applying Cramer's moderate deviation (see, e.g., Theorem 2.13 of [10]) with centered and scaled random variable $A_{n}^{-2}\left[\left(\xi_{j}-\mu_{j}\right)^{2} / \sigma_{j}^{2}-1\right]$ and deviation $c \sqrt{\log N}$, together with (15) and union bound. 
For the third inequality, we can re-write the off-diagonal error in $\Sigma$

$$
\hat{\Sigma}_{j, j^{\prime}}-\Sigma_{j, j^{\prime}}=n_{\mathrm{te}}^{-1} \sum_{i \in I_{\mathrm{te}}}\left(\xi_{j}^{(i)}-\mu_{j}\right)\left(\xi_{j^{\prime}}^{(i)}-\mu_{j^{\prime}}\right)-\left(\hat{\mu}_{j}-\mu_{j}\right)\left(\hat{\mu}_{j^{\prime}}-\mu_{j^{\prime}}\right)
$$

A similar argument as (18) shows that

$$
\left\|\left|\frac{\xi_{j}-\mu_{j}}{\sigma_{j}} \frac{\xi_{j^{\prime}}-\mu_{j^{\prime}}}{\sigma_{j^{\prime}}}-\Gamma_{j, j^{\prime}}\right|^{1 / 2}\right\|_{\psi_{1}} \leq A_{n}+(\log 2)^{-1} .
$$

Then the same argument leads to the desired result by combining the deviation bound on $\hat{\Sigma}-\Sigma$ and (16).

Proof of Theorem 4.1. Let $E$ denote the event that $\max _{j}\left|\hat{\mu}_{j}-\mu_{j}\right| / \sigma_{j} \leq c_{2} A_{n} \sqrt{\log N / n}$, $\max _{j}\left|\hat{\sigma}_{j} / \sigma_{j}-1\right| \leq c_{2} A_{n}^{2} \sqrt{\log N / n}$, and $\max _{j, j^{\prime}}\left|\hat{\Gamma}_{j, j^{\prime}}-\Gamma_{j, j^{\prime}}\right| \leq c_{2} A_{n}^{2} \sqrt{\log N / n}$. Lemma A.2 implies that under the condition of theorem we have $\mathbb{P}(E) \geq 1-3 N^{-1}$.

Let $\Lambda$ and $\hat{\Lambda}$ be the diagonal matrix corresponding to $\Sigma$ and $\hat{\Sigma}$, respectively. For any positive semidefinite matrix $\Sigma$, let $Z_{\Sigma}$ be an $N(0, \Sigma)$ random vector. For $\alpha \in(0,1)$, let $z(\alpha, \Sigma)$ be the upper $\alpha$ quantile of the maximum of $Z_{\Sigma}$.

For the first part of the theorem, by assumption we have $\max _{j} \mu_{j} / \sigma_{j} \leq \gamma_{n}$ with $\gamma_{n}=$ $r_{n} \sqrt{1 /(n \log N)}$. Recall that $\hat{p}$ is the bootstrap $p$-value given in Section 3.1.1. Here we ignore the bootstrap sampling variability and regard $\hat{p}$ as the limiting value when the bootstrap sample $B \rightarrow \infty$. We have

$$
\begin{aligned}
& \mathbb{P}(\hat{p} \leq \alpha) \\
= & \mathbb{P}\left[\sqrt{n_{\mathrm{te}}} \max _{j}\left(\frac{\hat{\mu}_{j}-\mu_{j}}{\hat{\sigma}_{j}}+\frac{\mu_{j}}{\hat{\sigma}_{j}}\right) \geq z(\alpha, \hat{\Gamma})\right] \\
\leq & \mathbb{P}\left[\sqrt{n_{\mathrm{te}}} \max _{j}\left(\frac{\hat{\mu}_{j}-\mu_{j}}{\hat{\sigma}_{j}}+\frac{\mu_{j}}{\hat{\sigma}_{j}}\right) \geq z(\alpha, \hat{\Gamma}), E\right]+\mathbb{P}\left(E^{c}\right) \\
\leq & \mathbb{P}\left[\sqrt{n_{\mathrm{te}}} \max _{j} \frac{\hat{\mu}_{j}-\mu_{j}}{\sigma_{j}} \geq z(\alpha, \hat{\Gamma})-c \sqrt{n} \gamma_{n}-c_{2}^{2} A_{n}^{3} \frac{\log N}{\sqrt{n}}, E\right]+\mathbb{P}\left(E^{c}\right) \\
\leq & \mathbb{P}\left[\sqrt{n_{\mathrm{te}}} \max _{j} \frac{\hat{\mu}_{j}-\mu_{j}}{\sigma_{j}} \geq z(\alpha+\delta, \Gamma)-c \sqrt{n} \gamma_{n}-c_{2}^{2} A_{n}^{3} \frac{\log N}{\sqrt{n}}, E\right]+\mathbb{P}\left(E^{c}\right) \\
\leq & \mathbb{P}\left[\max Z_{\Gamma} \geq z(\alpha+\delta, \Gamma)-c \sqrt{n} \gamma_{n}-c_{2}^{2} A_{n}^{3} \frac{\log N}{\sqrt{n}}\right]+C_{1} n^{-C_{2}}+3 N^{-1} \\
\leq & \alpha+\delta+\left(c \sqrt{n} \gamma_{n}+c_{2}^{2} A_{n}^{3} \frac{\log N}{\sqrt{n}}\right) 2(1+\sqrt{2 \log N})+C_{1} n^{-C_{2}}+3 N^{-1} \\
\leq & \alpha+c \max \left(\left(\frac{A_{n}^{4} \log ^{5} N}{n}\right)^{\frac{1}{6}}, r_{n},\left(\frac{A_{n}^{6} \log ^{3} N}{n}\right)^{\frac{1}{2}}, N^{-\left(C_{2} \wedge 1\right)}\right)
\end{aligned}
$$


where

- (19) follows from the concentration of $\hat{\mu}_{j}-\mu_{j}$ and $\hat{\sigma}_{j} / \sigma_{j}-1$, and the definition of $E$.

- (20) follows from the difference between maxima of Gaussian random vectors (Theorem 2 of [7]). Here $\delta=c A_{n}^{3}\left(\log ^{5 / 6} N\right) n^{-1 / 6}$ for some universal constant $c$.

- (21) follows from the Gaussian comparison of maxima of mean vectors (Corollary 2.1 of [5]) with the $\psi_{1}$ norm condition and $\log ^{7} N \leq c n^{1-c^{\prime}}$ for some constants $c, c^{\prime}$. Here $C_{1}$ and $C_{2}$ in (21) are universal constants involved in the Gaussian comparison result.

- (22) follows from the anti-concentration of maxima of Gaussian random vectors (Theorem 3 of [7]).

When $\mu_{j}=0$ for all $j$, the above argument also goes through in the other direction. In this case we have $\mathbb{P}(\hat{p} \leq \alpha)=\alpha+o(1)$. The first part of the theorem is complete.

For the second part. Let $j$ be such that $\frac{\mu_{j}}{\sigma_{j}} \geq r \sqrt{\frac{\log N}{n}}$. Then

$$
\begin{aligned}
\mathbb{P}(\hat{p} \leq \alpha) & =\mathbb{P}\left[\sqrt{n_{\mathrm{te}}} \max _{j^{\prime}} \frac{\hat{\mu}_{j^{\prime}}}{\hat{\sigma}_{j^{\prime}}} \geq z(\alpha, \hat{\Gamma})\right] \\
& \geq \mathbb{P}\left[\sqrt{n_{\mathrm{te}}} \frac{\hat{\mu}_{j}}{\hat{\sigma}_{j}} \geq z(\alpha, \hat{\Gamma})\right] \\
& \geq \mathbb{P}\left[\sqrt{n_{\mathrm{te}}} \frac{\hat{\mu}_{j}}{\hat{\sigma}_{j}} \geq z(\alpha, \hat{\Gamma}), E\right]
\end{aligned}
$$

On event $E$, we have

$$
\begin{aligned}
\frac{\hat{\mu}_{j}}{\hat{\sigma}_{j}} & =\left(\frac{\mu_{j}}{\sigma_{j}}+\frac{\hat{\mu}_{j}-\mu_{j}}{\sigma_{j}}\right)\left(\frac{\sigma_{j}}{\hat{\sigma}_{j}}-1+1\right) \\
& \geq\left(r-c_{2} A_{n}\right) \sqrt{\frac{\log N}{n}}\left(1-c_{2} A_{n}^{2} \sqrt{\frac{\log N}{n}}\right) \geq c \sqrt{\log N / n},
\end{aligned}
$$

for some constant $c>0$ when $n$ is large enough. Here the last inequality follows because by assumption we have $r \geq c^{\prime}\left(A_{n} \vee 1\right)$ for some large enough constant $c^{\prime}$.

On the other hand, using Mill's inequality and union bound we can directly verify that $z(\alpha, \hat{\Gamma}) \leq \sqrt{2(\log (M-1)-\log \alpha)} \leq 2 \sqrt{\log N}$ whenever $\alpha \geq n^{-1}$. Thus when $n$ is large enough we always have $\sqrt{n_{\mathrm{te}}} \hat{\mu}_{j} / \hat{\sigma}_{j} \geq z(\alpha, \hat{\Gamma})$ on $E$. As a result,

$$
\mathbb{P}(\hat{p} \leq \alpha) \geq \mathbb{P}(E) \geq 1-3 N^{-1} .
$$




\section{A.2 Proofs for Section 4.2}

Proof of Theorem 4.3. Let $\mathcal{M}=\{1,2, \ldots, M\}$. For any $m \in \mathcal{M}$, let $J_{m}$ be the subset of active variables in model $m$. Define $\mathcal{M}_{1}=\left\{m \in \mathcal{M}: J_{m^{*}} \backslash J_{m} \neq \emptyset\right\}$ be the candidate models missing at least one true variable, and $\mathcal{M}_{2}=\mathcal{M} \backslash\left(\mathcal{M}_{1} \cup\left\{m^{*}\right\}\right)$. We use " $a \lesssim b$ " to denote that $a \leq c b$ for some universal constant $c$.

By consistency of least square estimates we have $\sup _{m \in \mathcal{M}}\left\|\hat{\beta}_{m}-\beta_{m}\right\|_{2}=o_{P_{\mathrm{tr}}}(1)$, where $\beta_{m}=\Sigma_{J_{m}, J_{m}}^{-1} \mathbb{E}\left(X_{J_{m}}^{T} Y\right)$ is the population version, and the notation $o_{P_{\mathrm{tr}}}(\cdot)$ emphasizes that the randomness is on the training data $D_{\mathrm{tr}}$. In the following we overload the notation of $\hat{\beta}_{m}$ and $\beta_{m}$ by embedding them in $\mathbb{R}^{p}$, with zero values in the entries in $J_{m}^{c}$.

For two candidate models $m, m^{\prime}$, the difference of squared error is

$$
\begin{aligned}
\xi_{m, m^{\prime}} & =\left(Y-X^{T} \hat{\beta}_{m}\right)^{2}-\left(Y-X^{T} \hat{\beta}_{m^{\prime}}\right)^{2} \\
& =2 \epsilon X^{T}\left(\hat{\beta}_{m^{\prime}}-\hat{\beta}_{m}\right)+\left(X^{T}\left(\hat{\beta}_{m}-\beta\right)\right)^{2}-\left(X^{T}\left(\hat{\beta}_{m^{\prime}}-\beta\right)\right)^{2} .
\end{aligned}
$$

Now we show that for any $m \in \mathcal{M}_{1}$, we have $P\left(m \in \mathcal{A}_{n}\right) \rightarrow 0$.

Let $m^{\prime}=m^{*}$, then we have

$$
\begin{aligned}
\mathbb{E}\left(\xi_{m, m^{*}} \mid D_{\mathrm{tr}}\right) & =\left(\hat{\beta}_{m}-\beta\right)^{T} \Sigma\left(\hat{\beta}_{m}-\beta\right)+\left(\hat{\beta}_{m^{*}}-\beta\right)^{T} \Sigma\left(\hat{\beta}_{m^{*}}-\beta\right) \\
& \geq \beta_{\min }^{2} \lambda_{\min }+o_{P_{\mathrm{tr}}}(1)
\end{aligned}
$$

so that

$$
\hat{\mu}_{m, m^{*}} \geq \beta_{\min }^{2} \lambda_{\min }+o_{P}(1) .
$$

Since CVC uses a studentized test statistic, we still need to provide an upper bound for $\hat{\sigma}_{m, m^{*}}$. First we have, using the assumption that $X$ has finite fourth moment and $\epsilon X$ has finite second moment,

$$
\begin{aligned}
& \operatorname{Var}\left(\xi_{m, m^{*}} \mid D_{\mathrm{tr}}\right) \\
= & 4 \sigma^{2}\left(\hat{\beta}_{m}-\hat{\beta}_{m^{*}}\right)^{T} \Sigma\left(\hat{\beta}_{m}-\hat{\beta}_{m^{*}}\right)+\mathbb{E}\left[\left(\hat{\beta}_{m}+\hat{\beta}_{m^{*}}-2 \beta\right)^{T}\left(X X^{T}-\Sigma\right)\left(\hat{\beta}_{m}-\hat{\beta}_{m^{*}}\right)\right]^{2} \\
= & O_{P_{\mathrm{tr}}}\left(\sigma^{2} \lambda_{\max } \beta_{\max }^{2}+\beta_{\max }^{4} \lambda_{\max }^{2}\right)=O_{P_{\mathrm{tr}}}(1) .
\end{aligned}
$$

Thus

$$
\hat{\sigma}_{m, m^{*}}=O_{P}(1)
$$

Combining (25) and (27) we have

$$
\mathbb{P}\left[\sqrt{n_{\mathrm{te}}} \frac{\hat{\mu}_{m, m^{*}}}{\hat{\sigma}_{m, m^{*}}} \geq \sqrt{2 \log n}\right] \rightarrow 1
$$


As a consequence, the rejection probability of model $m$ approaches 1 as $n$ increases.

Now consider $m \in \mathcal{M}_{2}$. For brevity we denote $J_{m}$ as $J$ and $J_{m^{*}}$ as $J^{*}$. Let $\vec{\epsilon}$ denote the realized vector of $\epsilon$ in $D_{\mathrm{tr}}, X_{J}$ denote the training data columns of $X$ in $J$, and $\Sigma_{J}$ denote the corresponding population covariance matrix.

$$
\begin{aligned}
& \mathbb{E}\left(\left[X^{T}\left(\hat{\beta}_{m}-\beta\right)\right]^{2} \mid D_{\mathrm{tr}}\right) \\
&=\vec{\epsilon}^{T} X_{J}\left(X_{J}^{T} X_{J}\right)^{-1} \Sigma_{J}\left(X_{J, \mathrm{tr}}^{T} X_{J}\right)^{-1} X_{J}^{T} \vec{\epsilon} \\
&=\vec{\epsilon}^{T} X_{J}\left(X_{J}^{T} X_{J}\right)^{-1}\left[\Sigma_{J}-\left(X_{J}^{T} X_{J} / n_{\mathrm{tr}}\right)\right]\left(X_{J}^{T} X_{J}\right)^{-1} X_{J}^{T} \vec{\epsilon} \\
& \quad+n_{\mathrm{tr}}^{-1} \vec{\epsilon}^{T} X_{J}\left(X_{J}^{T} X_{J}\right)^{-1}\left(X_{J}^{T} X_{J}\right)\left(X_{J}^{T} X_{J}\right)^{-1} X_{J}^{T} \vec{\epsilon} \\
&=\vec{\epsilon}^{T} X_{J}\left(X_{J}^{T} X_{J}\right)^{-1}\left[\Sigma_{J}-\left(X_{J}^{T} X_{J} / n_{\mathrm{tr}}\right)\right]\left(X_{J}^{T} X_{J}\right)^{-1} X_{J}^{T} \vec{\epsilon} \\
& \quad+n_{\mathrm{tr}}^{-1} \vec{\epsilon}^{T} X_{J}\left(X_{J}^{T} X_{J}\right)^{-1} X_{J}^{T} \vec{\epsilon}
\end{aligned}
$$

where $P_{J}$ is the projection matrix of the linear subspace spanned by the columns of $X_{J}$.

Therefore

$$
n_{\mathrm{tr}} \mathbb{E}\left(\xi_{m, m^{*}} \mid D_{\mathrm{tr}}\right)=\vec{\epsilon}^{T}\left(P_{J}-P_{J^{*}}\right) \vec{\epsilon}+R_{n}
$$

where

$$
R_{n}=n_{\mathrm{tr}} \vec{\epsilon}^{T} X_{J}\left(X_{J}^{T} X_{J}\right)^{-1}\left[\Sigma_{J}-\left(X_{J}^{T} X_{J} / n_{\mathrm{tr}}\right)\right]\left(X_{J}^{T} X_{J}\right)^{-1} X_{J}^{T}
$$

Since $P_{J}-P_{J^{*}}$ is a projection matrix, we can write $P_{J}-P_{J^{*}}=\sum_{l=1}^{L} v_{l} v_{l}^{T}$, where $L \geq 1$ is the number of irrelevant variables in model $J$, and $v_{1}, \ldots, v_{L}$ are orthonormal vectors in $\mathbb{R}^{n_{\text {tr }}}$. Thus $\vec{\epsilon}^{T}\left(P_{J}-P_{J^{*}}\right) \vec{\epsilon} \geq\left(v_{1}^{T} \vec{\epsilon}\right)^{2}$. Note that $\left\|v_{1}\right\|_{\infty} \leq \max _{1 \leq i \leq n} H_{i i}^{1 / 2}$ where $H$ is the hat matrix with all columns of data. Let $r_{n}$ be a positive sequence such that $r_{n} \uparrow \infty$ and $r_{n}=o\left(n^{1 / 4}\right)$, then we have

$$
\begin{aligned}
& \mathbb{P}\left[\mathbb{E}\left(\xi_{m, m^{*}} \mid D_{\mathrm{tr}}\right)<0\right] \leq \mathbb{P}\left[\left(v_{1}^{T} \vec{\epsilon}\right)^{2}+R_{n}<0\right] \\
& \leq \mathbb{P}\left[\left|v_{1}^{T} \vec{\epsilon}\right|<\left|R_{n}\right|^{1 / 2}\right] \\
& \leq \mathbb{P}\left[\left|v_{1}^{T} \vec{\epsilon}\right|<r_{n}^{1 / 2} n^{-1 / 4}\right]+\mathbb{P}\left[\left|R_{n}\right|>r_{n} n^{-1 / 2}\right] \\
& \leq \mathbb{P}\left[\left|v_{1}^{T} \vec{\epsilon}\right|<r_{n}^{1 / 2} n^{-1 / 4} \mid\left\|v_{1}\right\|_{\infty} \leq 2 r_{n}^{1 / 2} n^{-1 / 4}\right] \\
& \quad+\mathbb{P}\left[\left\|v_{1}\right\|_{\infty}>2 r_{n}^{1 / 2} n^{-1 / 4}\right]+\mathbb{P}\left[\left|R_{n}\right|>r_{n} n^{-1 / 2}\right] \\
&=I+I I+I I I .
\end{aligned}
$$

For term $I$, we use anti-concentration inequality. Because $\epsilon$ has mean zero and positive variance, there exist constants $L>0$ and $\eta \in(0,1)$, such that $\sup _{t} P(\epsilon \in[t, t+L]) \leq 1-\eta$. 
Let $v_{1}=\left(v_{1, j}: 1 \leq j \leq n\right)$. If $v_{1, j} \neq 0$, then $\sup _{t} P\left(v_{1, j} \epsilon_{j} \in\left[t, t+\left|v_{1, j}\right| L\right]\right) \leq 1-\eta$. Now applying the anti-concentration inequality for sums of independent random variables (Theorem 1 of [25]), we have, when $\left\|v_{1}\right\|_{\infty} \leq 2 r_{n}^{1 / 2} n^{-1 / 4}$,

$$
\begin{aligned}
& \mathbb{P}\left(\left|v_{1}^{T} \vec{\epsilon}\right| \leq r_{n}^{1 / 2} n^{-1 / 4} \mid v_{1}\right) \\
\lesssim & \frac{r_{n}^{1 / 2} n^{-1 / 4}}{\sqrt{\sum_{j: v_{1, j} \neq 0} v_{1, j}^{2} L^{2}(1-\eta)}} \lesssim r_{n}^{1 / 2} n^{-1 / 4}=o(1) .
\end{aligned}
$$

For term $I I$, we have

$$
\mathbb{P}\left[\left\|v_{1}\right\|_{\infty}>2 r_{n}^{1 / 2} n^{-1 / 4}\right] \leq \mathbb{P}\left[\max _{i} H_{i i}>4 r_{n} n^{-1 / 2}\right]=o(1)
$$

where the last equation follows from the assumption that $\max _{i} H_{i i}=o_{P}\left(n^{-1 / 2}\right)$.

For term $I I I$, we have $I I I=o(1)$ since $r_{n} \rightarrow \infty$ and $R_{n}=O_{P}\left(n^{-1 / 2}\right)$, according to the assumption that $\epsilon X$ has finite second moment and $X$ has finite fourth moment.

Now we have shown that

$$
\mathbb{P}\left[\mathbb{E}\left(\xi_{m, m^{*}} \mid D_{\text {tr }}\right)<0\right]=o(1), \quad \forall m \in \mathcal{M}_{2} .
$$

Combining (24) and (29) we have

$$
\mathbb{P}\left[\sup _{m \neq m^{*}} \mathbb{E}\left(\xi_{m^{*}, m} \mid D_{\text {tr }}\right)>0\right]=o(1) .
$$

Now conditioning on the event $\sup _{m \neq m^{*}} \mathbb{E}\left(\xi_{m^{*}, m} \mid D_{\text {tr }}\right)<0$, we have

$$
\begin{aligned}
& \mathbb{P}\left[\sup _{m \neq m^{*}} \sqrt{n_{\mathrm{te}}} \frac{\hat{\mu}_{m^{*}, m}}{\hat{\sigma}_{m^{*}, m}} \geq z\left(\alpha_{n}, \hat{\Gamma}\right)\right] \\
\leq & \mathbb{P}\left[\sup _{m \neq m^{*}} \sqrt{n_{\mathrm{te}}} \frac{\hat{\mu}_{m^{*}, m}-\mu_{m^{*}, m}}{\hat{\sigma}_{m^{*}, m}} \geq z\left(\alpha_{n}, \hat{\Gamma}\right)\right] \\
= & o(1),
\end{aligned}
$$

where the last step we use the fact that $\sqrt{n_{\mathrm{te}}} \frac{\hat{\mu}_{m^{*}, m}-\mu_{m^{*}, m}}{\hat{\sigma}_{m^{*}, m}}=O_{P_{\mathrm{te}}}(1)$ according to the selfnormalized moderate deviation result (Theorem 7.4 of [10]), which holds since by condition B2 $\xi_{m^{*}, m}$ has finite third moment provided that $\hat{\beta}_{J_{m}^{*}}$ and $\hat{\beta}_{J_{m}}$ are bounded (with probability $\left.1-o_{P_{\mathrm{tr}}}(1)\right)$. 
Proof of Corollary 4.4. For given $v \in\{1,2, \ldots, V\}$, we treat $I_{v}$ as $I_{\text {te }}$ and $I_{-v}$ as $I_{\text {tr }}$. Using the same reasoning as from (24) to (27) on the $v$ th fold and then using union bound over $v=1, \ldots, V$, we have, if $m \in \mathcal{M}_{1}$, then with probability $1-o(1)$

$$
\inf _{v=1, \ldots, V} \frac{\hat{\mu}_{m, m^{*}}^{(v)}}{\hat{\sigma}_{m, m^{*}}^{(v)}} \geq c_{3}
$$

where $c_{3}>0$ is a constant depending on the distribution of $(X, \epsilon)$.

As a result,

$$
\frac{\hat{\mu}_{m, m^{*}}}{\hat{\sigma}_{m, m^{*}}}=\frac{\frac{1}{V} \sum_{v=1}^{V} \hat{\mu}_{m, m^{*}}^{(v)}}{\sqrt{\frac{1}{V} \sum_{v=1}^{V}\left(\hat{\sigma}_{m, m^{*}}^{(v)}\right)^{2}}} \geq \frac{\frac{1}{V} c_{3} \sum_{v=1}^{V} \hat{\sigma}_{m, m^{*}}^{(v)}}{\sqrt{\frac{1}{V} \sum_{v=1}^{V}\left(\hat{\sigma}_{m, m^{*}}^{(v)}\right)^{2}}} \geq \frac{c_{3}}{\sqrt{V}}
$$

When $n$ is large so that $c_{3} \sqrt{n / V} \geq \sqrt{2 \log N}$, then (let $\hat{\Gamma}$ be the sample correlation matrix of $\left.\left[\xi_{m, j}^{(i)}-\hat{\mu}_{m, j}^{\left(v_{i}\right)}: j \neq m\right]_{i=1, \ldots, n}\right)$

$$
\begin{aligned}
\mathbb{P}\left(\hat{p}_{\mathrm{cv}, m} \leq \alpha\right) & \geq \mathbb{P}\left[\sqrt{n} \frac{\hat{\mu}_{m, m^{*}}}{\hat{\sigma}_{m, m^{*}}} \geq z\left(\alpha_{n}, \hat{\Gamma}\right)\right] \geq P\left[\sqrt{n} \frac{\hat{\mu}_{m, m^{*}}}{\hat{\sigma}_{m, m^{*}}} \geq \sqrt{2 \log N}\right] \\
& \geq P\left[\sqrt{n} \frac{\hat{\mu}_{m, m^{*}}}{\hat{\sigma}_{m, m^{*}}} \geq c_{3} \sqrt{n / V}\right]=1-o(1) .
\end{aligned}
$$

Next we prove $\mathbb{P}\left(m^{*} \in \mathcal{A}_{\mathrm{cv}}\right) \rightarrow 1$. Applying the same argument as in the proof of Theorem 4.3 to each fold we have

$$
\mathbb{P}\left(\mu_{m^{*}, m}^{(v)}<0, \forall m \in \mathcal{M} \backslash\left\{m^{*}\right\}\right)=1-o(1) .
$$

Conditioning on this event, and using the same reasoning as in (30) we have

$$
\begin{aligned}
& \mathbb{P}\left[\sqrt{n} \frac{\hat{\mu}_{m^{*}, m}^{(v)}}{\hat{\sigma}_{m^{*}, m}^{(v)}} \geq z\left(\alpha_{n}, \hat{\Gamma}\right)\right] \\
\leq & P\left[\sqrt{n} \frac{\hat{\mu}_{m^{*}, m}^{(v)}-\mu_{m^{*}, m}^{(v)}}{\hat{\sigma}_{m^{*}, m}^{(v)}} \geq z\left(\alpha_{n}, \hat{\Gamma}\right)\right] \\
= & o(1),
\end{aligned}
$$

for all $m \neq m^{*}$ and $1 \leq v \leq V$. Finally using union bound we have

$$
\begin{aligned}
\mathbb{P}\left(m^{*} \notin \mathcal{A}_{\mathrm{cv}}\right) & =\mathbb{P}\left[\hat{p}_{\mathrm{cv}, m^{*}} \geq z\left(\alpha_{n}, \hat{\Gamma}\right)\right] \\
& =\mathbb{P}\left[\sup _{m \neq m^{*}} \sqrt{n} \frac{\hat{\mu}_{m^{*}, m}}{\hat{\sigma}_{m^{*}, m}} \geq z\left(\alpha_{n}, \hat{\Gamma}\right)\right]
\end{aligned}
$$




$$
\begin{aligned}
& \leq \mathbb{P}\left[\sup _{m \neq m^{*}} \sup _{1 \leq v \leq V} \sqrt{n} \frac{\hat{\mu}_{m^{*}, m}^{(v)}}{\hat{\sigma}_{m^{*}, m}^{(v)}} \geq z\left(\alpha_{n}, \hat{\Gamma}\right)\right] \\
& =o(1) .
\end{aligned}
$$

\section{References}

[1] David M Allen. The relationship between variable selection and data agumentation and a method for prediction. Technometrics, 16(1):125-127, 1974.

[2] Peter J Bickel, Ya'acov Ritov, and Alexandre B Tsybakov. Simultaneous analysis of lasso and dantzig selector. The Annals of Statistics, pages 1705-1732, 2009.

[3] Sourav Chatterjee and Jafar Jafarov. Prediction error of cross-validated lasso. arXiv preprint arXiv:1502.06291, 2015.

[4] Kehui Chen and Jing Lei. Network cross-validation for determining the number of communities in network data. Journal of the American Statistical Association, (just-accepted), 2016.

[5] Victor Chernozhukov, Denis Chetverikov, and Kengo Kato. Gaussian approximations and multiplier bootstrap for maxima of sums of high-dimensional random vectors. The Annals of Statistics, 41(6):2786-2819, 2013.

[6] Victor Chernozhukov, Denis Chetverikov, and Kengo Kato. Testing many moment inequalities. arXiv preprint arXiv:1312.7614, 2013.

[7] Victor Chernozhukov, Denis Chetverikov, and Kengo Kato. Comparison and anticoncentration bounds for maxima of gaussian random vectors. Probability Theory and Related Fields, 162(1-2):47-70, 2015.

[8] Denis Chetverikov and Zhipeng Liao. On cross-validated lasso. arXiv preprint arXiv:1605.02214, 2016.

[9] Arnak S Dalalyan, Mohamed Hebiri, Johannes Lederer, et al. On the prediction performance of the lasso. Bernoulli, 23(1):552-581, 2017.

[10] Victor H de la Peña, Tze Leung Lai, and Qi-Man Shao. Self-normalized processes: Limit theory and Statistical Applications. Springer Science \& Business Media, 2008.

[11] Bradley Efron, Trevor Hastie, Iain Johnstone, and Robert Tibshirani. Least angle regression. The Annals of statistics, 32(2):407-499, 2004.

[12] Yingying Fan and Cheng Yong Tang. Tuning parameter selection in high dimensional penalized likelihood. Journal of the Royal Statistical Society: Series B (Statistical Methodology), 75(3):531-552, 2013. 
[13] Yang Feng and Yi Yu. Consistent cross-validation for tuning parameter selection in high-dimensional variable selection. arXiv preprint arXiv:1308.5390, 2013.

[14] Davide Ferrari and Yuhong Yang. Confidence sets for model selection by f-testing. Statistica Sinica, pages 1637-1658, 2015.

[15] Seymour Geisser. The predictive sample reuse method with applications. Journal of the American Statistical Association, 70(350):320-328, 1975.

[16] Peter R Hansen, Asger Lunde, and James M Nason. The model confidence set. Econometrica, 79(2):453-497, 2011.

[17] Darren Homrighausen and Daniel J McDonald. Risk-consistency of cross-validation with lasso-type procedures. arXiv preprint arXiv:1308.0810, 2013.

[18] Francis KC Hui, David I Warton, and Scott D Foster. Tuning parameter selection for the adaptive lasso using eric. Journal of the American Statistical Association, 110(509):262-269, 2015.

[19] Jiming Jiang, J Sunil Rao, Zhonghua Gu, and Thuan Nguyen. Fence methods for mixed model selection. The Annals of Statistics, 36(4):1669-1692, 2008.

[20] Jing Lei, Max G'Sell, Alessandro Rinaldo, Ryan J Tibshirani, and Larry Wasserman. Distribution-free predictive inference for regression. Journal of the American Statistical Association, to appear, 2017.

[21] Ker-Chau Li. Asymptotic optimality for cp, cl, cross-validation and generalized crossvalidation: discrete index set. The Annals of Statistics, pages 958-975, 1987.

[22] Chinghway Lim and Bin Yu. Estimation stability with cross-validation (escv). Journal of Computational and Graphical Statistics, 25(2):464-492, 2016.

[23] Nicolai Meinshausen and Bin Yu. Lasso-type recovery of sparse representations for high-dimensional data. The Annals of Statistics, pages 246-270, 2009.

[24] Art B Owen and Patrick O Perry. Bi-cross-validation of the svd and the nonnegative matrix factorization. The annals of applied statistics, pages 564-594, 2009.

[25] Boris Alekseevich Rogozin. On the increase of dispersion of sums of independent random variables. Theory of Probability \& Its Applications, 6(1):97-99, 1961.

[26] Jun Shao. Linear model selection by cross-validation. Journal of the American statistical Association, 88(422):486-494, 1993.

[27] Mervyn Stone. Cross-validatory choice and assessment of statistical predictions. Journal of the Royal Statistical Society. Series B (Methodological), pages 111-147, 1974. 
[28] Ryan J Tibshirani and Robert Tibshirani. A bias correction for the minimum error rate in cross-validation. The Annals of Applied Statistics, pages 822-829, 2009.

[29] Alexandre B Tsybakov. Introduction to nonparametric estimation, 2009.

[30] Sara van de Geer and Johannes Lederer. The bernstein-orlicz norm and deviation inequalities. Probability Theory and Related Fields, 157(1-2):225-250, 2013.

[31] Sara A Van de Geer. High-dimensional generalized linear models and the lasso. The Annals of Statistics, pages 614-645, 2008.

[32] Aad W van der Vaart and Jon A Wellner. Weak Convergence and Empirical Processes. Springer, 1996.

[33] Tao Wang and Lixing Zhu. Consistent tuning parameter selection in high dimensional sparse linear regression. Journal of Multivariate Analysis, 102(7):1141-1151, 2011.

[34] Larry Wasserman and Kathryn Roeder. High dimensional variable selection. Annals of statistics, 37(5A):2178, 2009.

[35] Yuhong Yang. Consistency of cross validation for comparing regression procedures. The Annals of Statistics, pages 2450-2473, 2007.

[36] Ping Zhang. Model selection via multifold cross validation. The Annals of Statistics, pages 299-313, 1993. 